\title{
On catalyzed vacuum decay around a radiating black hole and the crisis of the electroweak vacuum
}

\author{
Takumi Hayashi, ${ }^{a, b}$ Kohei Kamada, ${ }^{b}$ Naritaka Oshita ${ }^{c}$ and Jun'ichi Yokoyama ${ }^{a, b, d, e}$ \\ ${ }^{a}$ Department of Physics, Graduate School of Science, The University of Tokyo, \\ Hongo 7-3-1 Bunkyo-ku, Tokyo 113-0033, Japan \\ ${ }^{b}$ Research Center for the Early Universe (RESCEU), Graduate School of Science, \\ The University of Tokyo, Hongo 7-3-1 Bunkyo-ku, Tokyo 113-0033, Japan \\ ${ }^{c}$ Perimeter Institute, 31 Caroline St., Waterloo, Ontario, N2L 2Y5, Canada \\ ${ }^{d}$ Kavli Institute for the Physics and Mathematics of the Universe (Kavli IPMU), UTIAS, WPI, \\ The University of Tokyo, Kashiwa, Chiba 277-8568, Japan \\ e Trans-scale Quantum Science Institute, The University of Tokyo, \\ Hongo 7-3-1 Bunkyo-ku, Tokyo 113-0033, Japan \\ E-mail: takumi_hayashi@resceu.s.u-tokyo.ac.jp, \\ kohei.kamada@resceu.s.u-tokyo.ac.jp, noshita@perimeterinstitute.ca, \\ yokoyama@resceu.s.u-tokyo.ac.jp
}

ABSTRACT: False vacuum decay is a key feature in quantum field theories and exhibits a distinct signature in the early Universe cosmology. It has recently been suggested that the false vacuum decay is catalyzed by a black hole $(\mathrm{BH})$, which might cause the catastrophe of the Standard Model Higgs vacuum if primordial BHs are formed in the early Universe. We investigate vacuum phase transition of a scalar field around a radiating $\mathrm{BH}$ with taking into account the effect of Hawking radiation. We find that the vacuum decay rate slightly decreases in the presence of the thermal effect since the scalar potential is stabilized near the horizon. However, the stabilization effect becomes weak at the points sufficiently far from the horizon. Consequently, we find that the decay rate is not significantly changed unless the effective coupling constant of the scalar field to the radiation is extremely large. This implies that the change of the potential from the Hawking radiation does not help prevent the Standard Model Higgs vacuum decay catalyzed by a $\mathrm{BH}$.

KEYwords: Black Holes, Solitons Monopoles and Instantons

ARXIV EPRINT: 2005.12808 


\section{Contents}

1 Introduction 1

2 Static bounce around a "zero-temperature" BH 4

2.1 General discussion on the bounce and vacuum decay rate catalyzed by $\mathrm{BH} 4$

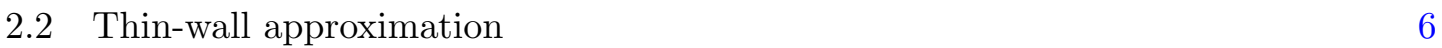

2.3 Connecting the parameters of thin-wall bubbles with the effective potential 11

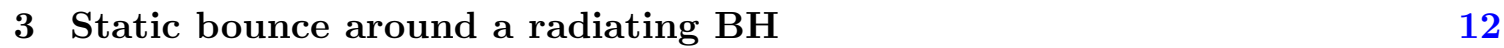

$\begin{array}{lll}3.1 & \text { Bounce solution around a radiating } \mathrm{BH} & 14\end{array}$

3.2 Thin-wall approximation of the bounce around a radiating BH 16

4 Implication to the Higgs instability 18

5 Conclusions and discussion $\quad 21$

\section{Introduction}

Vacuum phase transition is one of the most important phenomenon in the early universe predicted by quantum field theory. If our Universe is in a metastable state, namely a local minimum of a potential, quantum tunneling to the global minimum may take place. It includes a series of physical processes such as vacuum bubble nucleation. Such bubbles expand and collide with each other, which may become an important source of gravitational wave background $[1,2]$. Such metastable vacua often appear in various models of particle physics and string theories [3-5], and the most familiar example is the instability of electroweak vacuum [6-9]. The up-to-date measurements of the Higgs mass and top quark mass suggest that the effective potential of the Standard Model (SM) Higgs field develops a negative value at the scale higher than $10^{11} \mathrm{GeV}$ at their median values $[10,11]$, if there are not any corrections from the physics beyond the SM and quantum gravity. If the electroweak vacuum we live were unstable under such a phase transition with a considerable rate, our very existence would be in danger.

The rate of vacuum tunneling associated with such instabilities can be estimated by using the Euclidean path integral technique [12, 13], and subsequently gravitational effects were incorporated in ref. [14]. Applying it to the case of the Higgs instability, fortunately, the probability that our Universe undergoes the phase transition in the cosmic age is about $O\left(10^{-600}\right)[15,16]$. It is also sufficiently stable against the thermal phase transition regardless of the reheating temperature of the Universe $[9,17]$. The inflationary fluctuation is problematic especially for high-scale inflation, but several resolution has been proposed [18-20]. See also recent discussions on the SM Higgs vacuum stability in the 
inflationary Universe [21-23]. However, these works assume the homogeneity of the initial vacuum state and the inhomogeneity of the Universe is not taken into account.

It was found that the existence of spatial inhomogeneities greatly changes the result of the tunneling calculation, which was pioneered by Steinhardt [24] and Hiscock [25]. Hiscock first discussed the bubble nucleation around a non-rotating $\mathrm{BH}$ and showed that it can enhance the decay rate [25]. Gregory et al. refined and generalized his analysis [26-29] and pointed out that a very small $\mathrm{BH}$ greatly induces the phase transition process (see also [30]). Recently the study of catalyzing effects on cosmological vacuum decay was extended to the cases of spinning BHs [31] and other various cosmological impurities [32-35].

Cosmological creation of primordial black holes (PBHs) has been of interest for a long time to explain dark matter [36-38] and recently for the binary BHs [39] detected by the LIGO and VIRGO [40]. The density perturbations in the early universe could also lead to small-mass PBHs which have evaporated by today. Since those PBHs may play the role of catalysts for the Higgs vacuum decay, cosmological parameters relevant to the PBH formation may be constrained by the parameters of the Higgs potential or vice versa [41]. The footprint of PBH evaporation could remain in stochastic gravitational waves [42], which may be detected by near-future GW observations. Therefore, the catalyzing effect of BHs are very important not only in the particle physics but also in the early cosmology.

According to the studies in refs. [26-28], the formation of such small BHs might be dangerous for our Universe since they can induce the SM Higgs vacuum phase transition at the end stage of the evaporation. However, the thermal effects of small BHs could be non-negligible since the Hawking temperature is proportional to the inverse of its mass, and it has been an open question whether the thermal effects of small BHs would stabilize the present Higgs vacuum state or not. ${ }^{1}$

In order to investigate this phenomenon in more detail, it is important to construct the bounce solution based on the effective potential including thermal (quantum) corrections appropriately. Indeed, since we are interested in tiny evaporating BHs, we need to use the effective action that describes radiating BHs. In particular, the Hawking radiation emitted from the BHs might stabilize the Higgs potential or make the Higgs potential barrier high enough to prevent the transition from the false to true vacuum state [43]. Such a backreaction is quite important when we discuss the phase transition in thermal plasma [17, 44], where quantum fields obtain large vacuum polarization associated with the high temperature. In this paper, we take into account this effect on the analysis of the vacuum phase transition around a radiating $\mathrm{BH}$ and try to improve the evaluation of the transition rate.

The crucial point in our analysis is the choice of the vacuum state in the Schwarzschild spacetime. We are interested in the cosmological application of the transition process around a BH. Therefore, we here consider a vacuum state around a gravitationally collapsed $\mathrm{BH}$ which has no past horizon that separates the regions I and III in the Penrose diagram

\footnotetext{
${ }^{1}$ For example, ref. [43] argues that the thermal effects of Hawking radiation would stabilize the present Higgs vacuum state and a small BH does not play a role of a catalyst for the Higgs vacuum decay. However, their approach differs from the Euclidean path integral, utilized in [26], and the stochastic fluctuation of the Higgs field is assumed although the background is Schwarzschild spacetime.
} 


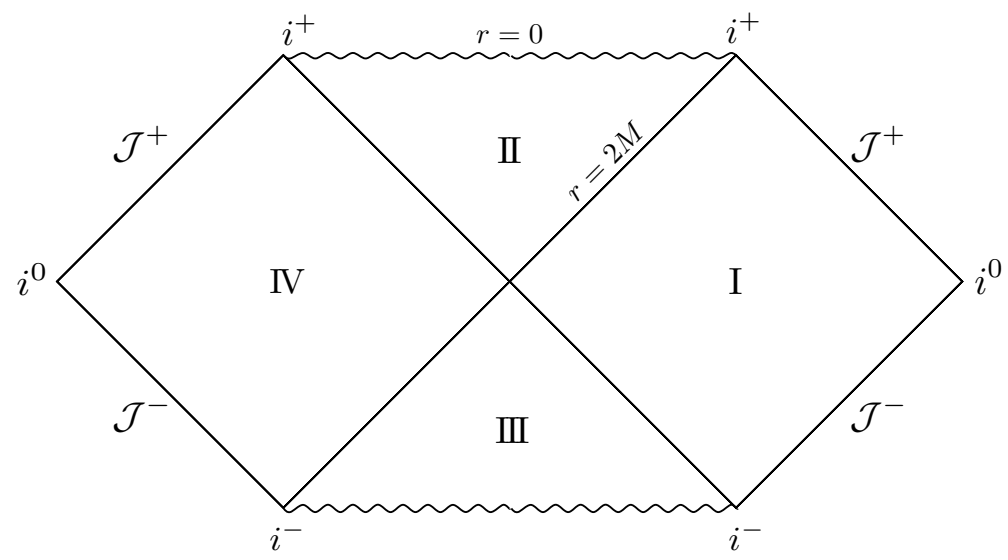

Figure 1. The Penrose diagram of maximally extended Schwarzschild spacetime. Region I and II are the outside and the inside of the event horizon respectively, and region III is the white hole interior. Region IV is the mirror domain of region I. Physically relevant regions to a BH formed by gravitational collapse are Region I and II.

(figure 1). In this case, the state of quantum fields can be modeled by the Unruh vacuum state [45] that leads to outgoing energy flux of vacuum fluctuations and the evaporation process of BHs. On the other hand, the Hartle-Hawking vacuum state [46] describes a thermal equilibrium state around a $\mathrm{BH}$ and it does not evaporate. ${ }^{2}$ An important feature of the Higgs potential thermally corrected in the Unruh vacuum state is that the thermal correction becomes weaker at a larger distance from the horizon [48]. As a result, we will show that the thermal effects do not prevent the catalyst effect of BHs since the Hawking temperature is suppressed near a bubble wall unless there are many light scalar fields $\chi_{i}$ coupling to the metastable scalar field $\phi$ (e.g. Higgs field) as $\sim \sum_{i} \lambda_{i} \chi_{i}^{2} \phi^{2}$ with $\sum_{i} \lambda_{i} \gg 10^{3}$, where $\lambda_{i}$ is a coupling constant. This general result also holds in the case of the SM Higgs vacuum and hence the tiny PBH formation in the early Universe is still a threat of our Universe.

The organization of this paper is as follows. In the next section, we review the vacuum phase transition around a non-radiating $\mathrm{BH}$ following refs. [27, 28] and obtain simple expression of transition rate with the thin-wall approximation. In section 3 , we include the effect of Hawking radiation in the potential, calculate the bounce solution, and estimate the transition rate. In section 4, we apply this result to the case of Higgs vacuum instability and argue that a vacuum bubble nucleation due to the Higgs instability would be catalyzed by a $\mathrm{BH}$ even when the thermal effect of Hawking radiation is taken into account. Section 5 is devoted to conclusion and discussion.

We adopt the Planck units for simplicity; the Newton constant $G=1$, Planck mass $m_{\mathrm{pl}}=1$, and Planck length $\ell_{\mathrm{pl}}=1$. When $G$ explicitly appears in some equations or definitions, it is intended that $G$ denote the original mass dimension of physical quantities.

\footnotetext{
${ }^{2}$ There is a related work [47] that suggests the large enhancement of the transition rate can be interpreted as the thermal production of a bubble in the Hawking radiation in the Hartle-Hawking vacuum state (thermal equilibrium state).
} 


\section{Static bounce around a "zero-temperature" BH}

We start with the review of vacuum phase transition around a Schwarzschild BH following ref. [28]. Here we consider a scalar field $\phi$ with a potential that has two minima, $\phi=$ $\phi_{\mathrm{fv}}(=0)$ and $\phi=\phi_{\mathrm{tv}}$, where the former is a false vacuum with a vanishing potential energy density and the latter is a true vacuum with a negative potential energy density, and evaluate the phase transition rate from the false to true vacuum through the bubble nucleation around the $\mathrm{BH}$.

\subsection{General discussion on the bounce and vacuum decay rate catalyzed by BH}

Let us consider the following action

$$
S=\int_{\mathcal{M}} d^{4} x \sqrt{-g}\left(\frac{1}{16 \pi} \mathcal{R}-\frac{1}{2}\left(\partial_{\mu} \phi\right)^{2}-V(\phi)\right)+\frac{1}{8 \pi} \int_{\partial \mathcal{M}} K d S
$$

where $\mathcal{M}$ is the spacetime manifold with a $\mathrm{BH}, g$ is the determinant of the (Lorentzian) metric $g_{\mu \nu}$ with the convention $(-,+,+,+), \mathcal{R}$ is the Ricci scalar, and $K$ is the trace of the extrinsic curvature of the boundary of the spacetime. Since we consider the spacetime with a BH horizon, we explicitly include the Gibbons-Hawking-York boundary term. Assuming the spherical symmetry and staticity of the final configuration, a general metric is taken to be

$$
d s^{2}=-f(r) e^{2 \delta(r)} d t^{2}+f^{-1}(r) d r^{2}+r^{2} d \Omega_{2}^{2}, \quad f=1-\frac{2 \mu(r)}{r},
$$

where $d \Omega_{2}^{2}$ is the line elements on a unit sphere $S^{2}$. Solving the Einstein equation, together with the equation of motion for scalar fields, one can obtain the metric functions $\mu(r)$ and $\delta(r)$ and can determine a vacuum bubble configuration. On the other hand, the initial configuration is given by the Schwarzschild metric with $M_{+}$being the seed $\mathrm{BH}$ mass

$$
d s^{2}=-\left(1-\frac{2 M_{+}}{r}\right) d t^{2}+\left(1-\frac{2 M_{+}}{r}\right)^{-1} d r^{2}+r^{2} d \Omega_{2}^{2}
$$

In order to estimate the Euclidean action, let us implement the Wick rotation $t \rightarrow-i \tau$, and one obtains the Euclidean spacetime

$$
d s_{\mathrm{E}}^{2}=g_{\mathrm{E} \mu \nu} d x^{\mu} d x^{\nu}=f e^{2 \delta} d \tau^{2}+f^{-1} d r^{2}+r^{2} d \Omega_{2}^{2} .
$$

Then one can construct the bounce solutions on the Euclidean background. The existence of a $\mathrm{BH}$ reduces the maximal symmetry of bounce to $O(3)$ from $O(4)$. Therefore, we here consider time-independent static $O(3)$ symmetric solutions. We can still construct timedependent bounce solutions, but it has been shown that the least Euclidean action is given by the static one based on the analysis under the thin-wall condition [26]. Note that the bounce is static even after analytic continuation to Lorentzian bubble, but it is unstable under the perturbations and can easily expand so that the whole system quickly falls down to the (unwanted) true vacuum. Thus we regard the bubble nucleation rate, evaluated 
from the bounce action, as the vacuum decay rate of the system. The equation of motion for the scalar field and the Einstein equations are

$$
\begin{aligned}
& f \phi^{\prime \prime}+f^{\prime} \phi^{\prime}+\frac{2}{r} f \phi^{\prime}+\delta^{\prime} f \phi^{\prime}-\frac{\partial V}{\partial \phi}=0, \\
& \mu^{\prime}=4 \pi r^{2}\left(\frac{1}{2} f \phi^{\prime 2}+V\right), \\
& \delta^{\prime}=4 \pi r \phi^{\prime 2},
\end{aligned}
$$

where the prime denotes the derivative with respect to $r$. Substituting eq. (2.7) into eq. (2.5), one obtains

$$
f \phi^{\prime \prime}+f^{\prime} \phi^{\prime}+\frac{2}{r} f \phi^{\prime}+4 \pi f r \phi^{\prime 3}-\frac{\partial V}{\partial \phi}=0 .
$$

We require that the scalar field is in the false vacuum state at infinity and the asymptotic spacetime is the Schwarzschild spacetime:

$$
\mu(r) \rightarrow M_{+}, \quad \phi(r) \rightarrow \phi_{\mathrm{fv}}, \quad \phi^{\prime}(r)=0, \quad(r \rightarrow \infty),
$$

where $M_{+}$denotes the initial BH mass before the nucleation. We also impose the following boundary condition at the horizon $r=r_{h}$

$$
\mu(r) \rightarrow \mu_{-}, \quad \phi(r) \rightarrow \phi_{0} \quad\left(r \rightarrow r_{h}\right),
$$

where

$$
\mu_{-} \equiv \mu\left(r_{h}\right)=\frac{r_{h}}{2},
$$

and $\mu_{-}$and $\phi_{0}$ are determined by the shooting method so that the obtained solution satisfies the condition (2.9). In order for the solution to avoid the coordinate singularity at the horizon in eq. (2.5), the following condition should be satisfied at the same time

$$
\phi^{\prime}\left(r_{h}\right)=\frac{r_{h} \frac{\partial V}{\partial \phi}\left(\phi_{0}\right)}{1-8 \pi r_{h}^{2} V\left(\phi_{0}\right)} .
$$

One can calculate $\delta(r)$ after obtaining $\phi(r)$ from the integration of eq. (2.6) and (2.8), and its integration constant can be absorbed into the scale of $\tau$. Furthermore, we perform the coordinate transformation to improve the numerical behavior near the horizon as

$$
r^{*}=\int \frac{d r}{f(r)},
$$

which runs from the horizon $-\infty$ to the spacial infinity $\infty$. Consequently, the bounce equations, (2.6) and (2.8), become

$$
\begin{aligned}
& \frac{d^{2} \phi}{d r^{*}}+\frac{2 f}{r} \frac{d \phi}{d r^{*}}+\frac{4 \pi r}{f}\left(\frac{d \phi}{d r^{*}}\right)^{3}-f \frac{\partial V}{\partial \phi}=0, \\
& \frac{d \mu}{d r^{*}}=4 \pi r^{2}\left(\frac{1}{2}\left(\frac{d \phi}{d r^{*}}\right)^{2}+f V\right),
\end{aligned}
$$


and the boundary conditions at the horizon (eqs. (2.10) and (2.12)) become

$$
\mu\left(r^{*}\right) \rightarrow \mu_{-}, \quad \phi\left(r^{*}\right) \rightarrow \phi_{0}, \quad d \phi / d r^{*} \rightarrow 0 \quad\left(r^{*} \rightarrow-\infty\right) .
$$

The exponent of vacuum decay rate can be evaluated by the difference between the Euclidean action $S_{E}$ of the bounce and of the false vacuum solution before the transition, and the full form of the decay rate can be obtained as [26]

$$
\Gamma_{\mathrm{D}} \sim \sqrt{\frac{B}{2 \pi}} M_{+}^{-1} \exp (-B), \quad \text { with } \quad B=S_{E}\left[g_{E}, \phi\right]-S_{E}\left[g_{\mathrm{ESch}}, \phi_{\mathrm{fv}}\right]
$$

where $M_{+}$is initial BH mass and $g_{\text {Esch }}$ denotes the Euclideanized Schwarzschild metric, see eq. (2.3). $\left(\phi, g_{E}\right)$ represents the bounce solution obtained by solving the bounce equations. The dimensionless factor $\sqrt{B / 2 \pi}$ comes from the normalization factor of the zero mode around the bounce associated with the time translation symmetry. The dimensionful prefactor $M_{+}^{-1}$ is taken from the typical energy scale of the transition process [26]. For the static bounce solution, the bulk part of the action vanishes due to the Hamiltonian constraint and only the boundary part, which comes from the Gibbons-Hawking-York term, contributes to the action [26]. The boundary contribution reduces to $(-1)$ times the Bekenstein-Hawking entropy that can be derived without tuning the period of the Euclidean time (even with the conical singularity)

$$
S_{E}\left[g_{E}, \phi\right]=-\frac{\mathcal{A}}{4},
$$

where $\mathcal{A}$ is the horizon area [26]. Consequently the bounce action is determined to be

$$
B=4 \pi\left(M_{+}^{2}-\mu_{-}^{2}\right) .
$$

Since this expression generally holds for static bounce solutions, we will use the expression in the next section where we consider static bounce solutions with radiating BHs.

\subsection{Thin-wall approximation}

In principle we need to numerically solve the bounce equations (2.5), (2.6) and (2.7) for a given potential to see how the vacuum decay rate changes in the presence of a BH. However, when the thin-wall approximation is applicable, we can give an analytic investigation to show some qualitative and quantitative features of the $\mathrm{BH}$ catalyst effect.

In the thin-wall approximation, one can suppose that an infinitely-thin wall separates two different spacetimes and the scalar field can be represented by the step function at the wall. The system is, then, characterized by the tension of the wall $\sigma$, the bubble radius $R$, and the interior vacuum energy density, characterized by anti-de Sitter (AdS) radius $l \equiv \sqrt{3 /\left|8 \pi V\left(\phi_{\mathrm{tv}}\right)\right|}$, where the false vacuum energy density is assumed to be zero. The details of the effective potential barrier is "coarse-grained" in the tension parameter $\sigma$ as

$$
\sigma=\int_{\phi_{\mathrm{fv}}}^{\phi_{\mathrm{tv}}} d \phi \sqrt{2 V(\phi)} \sim V_{\mathrm{barrier}}^{1 / 2} \Delta \phi, \quad \Delta \phi \equiv\left|\phi_{\mathrm{tv}}-\phi_{\mathrm{fv}}\right|,
$$


where $V_{\text {barrier }}$ is the maximum value of effective potential between the false and true vacuum, and we suppose it is much larger than the potential energy difference, $V_{\text {barrier }} \gg$ $\Delta V \equiv\left|V\left(\phi_{\mathrm{fv}}\right)-V\left(\phi_{\mathrm{tv}}\right)\right|$. Thin-wall approximation is appropriate if the bubble-wall thickness is thinner than any relevant length scales, e.g. the bubble radius. While the bubble radius is determined by the balance between the energy contributions from the bulk $E_{\text {bulk }}$ and the wall $E_{\text {wall }}$. For a spherical bubble, we roughly estimate

$$
\left|E_{\text {bulk }}\right| \sim \frac{4 \pi}{3} R^{3} \Delta V, \quad E_{\text {wall }} \sim 4 \pi R^{2} \sigma \simeq 4 \pi V_{\text {barrier }}^{1 / 2} \Delta \phi R^{2},
$$

which give the bubble radius in terms of the potential parameters,

$$
R \sim \frac{V_{\text {barrier }}^{1 / 2} \Delta \phi}{\Delta V} .
$$

On the other hand the wall thickness is determined by the curvature of the potential top at $\phi=\phi_{\text {barrier }}, l_{\text {wall }} \sim m_{\text {barrier }}^{-1} \equiv \sqrt{V^{\prime \prime}\left(\phi_{\text {barrier }}\right)}$. Thus we see that the thin-wall approximation is appropriate when the energy difference between the false and true vacuum, $\Delta V$, is sufficiently small,

$$
\Delta V \ll V_{\text {barrier }}^{1 / 2} m_{\text {barrier }} \Delta \phi .
$$

In the following, we assume that eq. (2.23) holds and calculate the dynamics of a thinwall bubble which is consistent with the Einstein equation. To this end, we solve the Israel junction condition [49] with the $O(3)$ symmetry:

$$
K_{a b}^{(+)}-K_{a b}^{(-)}=8 \pi G\left(S_{a b}-\frac{1}{2} h_{a b} S\right),
$$

where $K_{a b}^{(+/-)}$is the extrinsic curvature outside/inside the bubble wall, $h_{a b}$ is the induced metric on the wall, and $S_{a b}=-\sigma h_{a b}$ is the energy momentum tensor of the wall. The trajectory of the bubble is given by

$$
X_{ \pm}^{\mu}=\left(\tau_{ \pm}(\eta), R(\eta), \theta, \phi\right),
$$

where $\eta$ is Euclidean proper time and $\tau_{+/-}$is the Schwarzschild time for the exterior/interior spacetime. Outside the bubble we take the scalar field is at the false vacuum and the metric is the Schwarzschild metric,

$$
\phi=\phi_{\mathrm{fv}}, \quad d s_{\mathrm{E}+}^{2}=f_{+} d \tau_{+}^{2}+f_{+}^{-1} d r_{+}^{2}+r^{2} d \Omega_{2}^{2}, \quad \text { with } \quad f_{+}(r)=1-\frac{2 M_{+}}{r},
$$

whereas inside the bubble we take the scalar field is at the true vacuum and the metric is the Schwarzschild-AdS metric,

$$
\phi=\phi_{\mathrm{tv}}, \quad d s_{\mathrm{E}-}^{2}=f_{-} d \tau_{-}^{2}+f_{-}^{-1} d r_{-}^{2}+r^{2} d \Omega_{2}^{2}, \quad \text { with } \quad f_{-}(r)=1-\frac{2 M_{-}}{r}+\frac{r^{2}}{l^{2}},
$$

where $M_{+}$and $M_{-}$are the $\mathrm{BH}$ masses before and after the bubble nucleation, respectively, and we set $r_{+}=r_{-}=R$ at the bubble wall. Note that the horizon and the $\mathrm{BH}$ mass inside the bubble are related as

$$
M_{-}=\frac{4}{l^{2}} \mu_{-}^{3}+\mu_{-}, \quad \mu_{-}=r_{h} / 2
$$


Then the $(\theta, \theta)$-component of the Israel junction condition reduces to

$$
f_{+}(R) \dot{\tau}_{+}-f_{-}(R) \dot{\tau}_{-}=-\frac{\bar{\sigma} R}{2}, \quad(\bar{\sigma} \equiv 8 \pi \sigma),
$$

where the dot represents the derivative with respect to $\eta$. Imposing the condition that the magnitude of the wall four-velocity to be the unity, one obtains

$$
f_{ \pm}(R) \dot{\tau}_{ \pm}^{2}+\frac{\dot{R}^{2}}{f_{ \pm}(R)}=1
$$

Using eq. (2.30), the Israel junction condition (2.29) reduces to

$$
\dot{R}^{2}=1-\left(\frac{\bar{\sigma}^{2}}{16}-\frac{1}{2 l^{2}}+\frac{1}{\bar{\sigma}^{2} l^{4}}\right) R^{2}-\left(M_{+}+M_{-}+\frac{4\left(M_{+}-M_{-}\right)}{\bar{\sigma}^{2} l^{2}}\right) \frac{1}{R}-\frac{4\left(M_{+}-M_{-}\right)^{2}}{\bar{\sigma}^{2} R^{4}} .
$$

By redefining the variables as

$$
\tilde{R}=\alpha R, \quad \tilde{\tau}=\alpha \tau, \quad \tilde{\eta}=\alpha \eta, \quad \text { with } \quad \alpha \equiv \frac{1-\bar{\sigma}^{2} l^{2} / 4}{\bar{\sigma} l^{2}},
$$

the equation for the wall position is rewritten as

$$
\frac{1}{2}\left(\frac{d \tilde{R}}{d \tilde{\lambda}}\right)^{2}+U(\tilde{R})=0, \quad 2 U(\tilde{R}) \equiv-1+\left(\tilde{R}+\frac{k_{2}}{\tilde{R}^{2}}\right)^{2}+\frac{k_{1}}{\tilde{R}}
$$

where

$$
k_{1}=2 \alpha M_{+}, \quad k_{2}=\frac{2 \alpha^{2}\left(M_{+}-M_{-}\right)}{\bar{\sigma}} .
$$

Here we consider the case $\bar{\sigma} l<1 / 2$. Indeed, it was shown that there is no any solutions that satisfy an appropriate junction condition for $\bar{\sigma} l>1 / 2$ [27].

The $O(3)$ static solution, which gives the highest decay rate, are obtained by requiring that there are parameter sets satisfying $U(\tilde{R})=U^{\prime}(\tilde{R})=0$. This determines the relation between the parameters $k_{1}$ and $k_{2}$

$$
\begin{aligned}
k_{1} & =k_{1}^{*}\left(k_{2}\right) \equiv-2 k_{2} \mp \frac{2}{9} \sqrt{1+81 k_{2}^{2}+Q_{+}-Q_{-}} \text {for } k_{2} \lessgtr-\frac{2}{3 \sqrt{3}} \\
\text { with } Q_{ \pm} & =\left( \pm\left(1+5\left(27 k_{2}\right)^{2}-\frac{\left(27 k_{2}\right)^{4}}{2}\right)+\frac{27 k_{2}}{2}\left(\left(27 k_{2}\right)^{2}+4\right)^{\frac{3}{2}}\right)^{\frac{1}{3}},
\end{aligned}
$$

as well as the position of the wall,

$$
\tilde{R}=\tilde{R}_{*} \equiv 2^{-\frac{2}{3}}\left(k_{1}^{*}+2 k_{2}+\sqrt{k_{1}^{*}+4 k_{1}^{*} k_{2}+36 k_{2}^{2}}\right)^{\frac{1}{3}} .
$$

Note that $k_{1}^{*}$ is positive for $k_{2}<4 / 27$.

From eq. (2.35) we can determine $M_{-}$(and hence $\mu_{-}$from eq. (2.28)) in terms of $\sigma$, $l$, and $M_{+}$. The bounce action is then determined by eq. (2.19). Note that the effective 
potential governing the position of the wall is concave, $W^{\prime \prime}(\tilde{R})<0$, in the Lorentzian picture, $W(\tilde{R}) \equiv-U(\tilde{R})$. Therefore, the static bubble is unstable and it may eventually collapse or expand after the nucleation. If the Hawking radiation that has outgoing flux interacts with the bubble wall, it may push the wall outward and the bubble would expand. Thus, as we have mentioned in the above, we expect that the nucleated bubbles are likely to expand to the spatial infinity, which is the catastrophe of the Universe, and hence we treat the bubble nucleation rate as the vacuum decay rate.

It is instructive to see the large and small- $M_{+}$limits of the solution. For $k_{2} \ll-1$ (large- $M_{+}$limit), we see $k_{1}^{*} \gg 1$ or $M_{+} \gg 1 / 2 \alpha$ so that

$$
k_{1}^{*} \simeq\left(-k_{2}\right)^{1 / 3}+\frac{1}{36\left(-k_{2}\right)^{1 / 3}}+\mathcal{O}\left(\left(-k_{2}\right)^{-2 / 3}\right), \quad R_{*} \simeq 2 M_{+}\left(1+\frac{1}{144 \alpha^{2} M_{+}^{2}}\right),
$$

which implies that the bubble is created near the horizon. From eqs. (2.19), (2.28), (2.34), and (2.38), then, we obtain the horizon mass inside the bubble and the bounce action as

$$
\begin{aligned}
& \mu_{-} \simeq\left(1-\bar{\sigma}^{2} l^{2} / 4\right)^{\frac{1}{3}} M_{+}, \\
& B \simeq 4 \pi\left(1-\left(1-\bar{\sigma}^{2} l^{2} / 4\right)^{\frac{2}{3}}\right) M_{+}^{2},
\end{aligned}
$$

at the first order in $M_{+}$.

For $k_{2} \sim 4 / 27$ (small- $M_{+}$limit), we see $k_{1} \ll 1$ or $M_{+} \ll 1 / 2 \alpha$, which leads to

$$
k_{1}^{*} \simeq 3\left(\frac{4}{27}-k_{2}\right), \quad R \simeq \frac{2}{3 \alpha}-\frac{M_{+}}{2},
$$

which means that the bubble wall is placed far from the horizon, and the configuration is close to an static $O(3)$ bubble without a BH. Combining eq. (2.41) with eqs. (2.19), (2.28), and (2.34), we obtain the asymptotic expressions

$$
\begin{aligned}
& \mu_{-} \simeq M_{+}-\frac{2 \bar{\sigma}^{3} l^{4}}{27\left(1-\bar{\sigma}^{2} l^{2} / 4\right)^{2}} \\
& B \simeq \frac{16 \pi \bar{\sigma}^{3} l^{4}}{27\left(1-\bar{\sigma}^{2} l^{2} / 4\right)^{2}} M_{+}+4 \pi\left(\frac{2 \bar{\sigma}^{3} l^{4}}{27\left(1-\bar{\sigma}^{2} l^{2} / 4\right)^{2}}\right)^{2} .
\end{aligned}
$$

For $\bar{\sigma} l \ll 1$, when the gravitational effect is small, the bounce action is further approximated as

$$
B \simeq \frac{16 \pi}{27} \bar{\sigma}^{3} l^{4} M_{+} .
$$

Figure 2 shows the bounce action as a function of the $\mathrm{BH}$ mass before the bubble nucleation $M_{+}$with $\sigma=3.42 \times 10^{-15}$ and $l=9.36 \times 10^{10}$.

Since we are interested in the bubble nucleation catalyzed by radiating BHs, the bubble nucleation rate evaluated in the above is meaningful only if it is larger than the $\mathrm{BH}$ evaporation rate $[50,51]$,

$$
\Gamma_{\mathrm{H}} \equiv \dot{M} / M \simeq 7.5 \times 10^{-5} g_{H} M_{+}^{-3}
$$




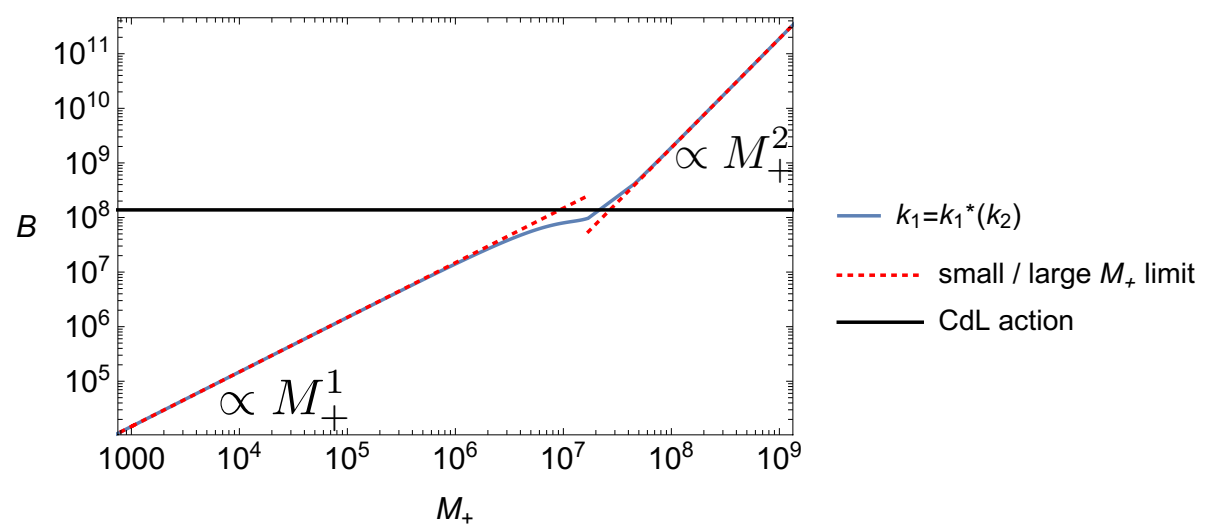

Figure 2. The bounce action dependence on initial BH mass $M_{+}$for $\bar{\sigma}=3.42 \times 10^{-15}$ and $l=9.36 \times 10^{10}$ which corresponds to the potential parameters (eq. (2.48)) $m=4.08 \times 10^{-6}$, $g=2.13, \lambda=1$. The blue line represents the bounce action obtained from the condition $k_{1}=k_{1}^{*}\left(k_{2}\right)$ (eq. (2.35)), and the red dotted lines the asymptotic forms given by eqs. (2.40) and (2.43). Black solid line means the CDL bounce action given by eq. (2.47). The bounce action of the $O(3) \times \mathrm{U}(1)$ bubble become significantly smaller than the CDL's one as $M_{+} \ll 10^{7}$.

where $g_{H}$ is the effective degrees of freedom emitted as the Hawking radiation,

$$
g_{H} \simeq \sum_{i} g_{s_{i}}, \quad \text { with } \quad g_{s}= \begin{cases}1 & s=0 \\ 0.55 & s=\frac{1}{2} \\ 0.22 & s=1 \\ 0.003 & s=2\end{cases}
$$

where $i$ denotes the particle species whose mass is lighter than Hawking temperature, and $s$ is the spin of the particle. Note that $g_{H} \simeq 60$ is obtained in the case of SM for a small BH satisfying $M \ll 10^{17}$.

Since the bounce action increases in proportion to the BH mass before the phase transition, $M_{+}$, the bubble nucleation rate becomes exponentially smaller for larger $M_{+}$, see eq. (2.17). Comparing it with eq. (2.45), which shows the decay of the $\mathrm{BH}$ evaporation rate proportional to a power law of $M_{+}$, we see that for smaller $M_{+}$, the bubble nucleation rate is larger than the $\mathrm{BH}$ evaporation rate. For example, for $\sigma \sim 10^{-15}, l \sim 10^{10}$, and $g_{H} \sim 60, \Gamma_{\mathrm{H}}<\Gamma_{\mathrm{D}}$ holds for $M_{+} \lesssim 10^{7}$. Though this discussion is limited to the thin-wall case, it has been found that even in the case when the thin-wall approximation does not hold (see eq. (2.23)), $\Gamma_{\mathrm{H}}<\Gamma_{\mathrm{D}}$ is likely to be satisfied for small $M_{+}$[28]. Note that for $M_{+}<1$, the $\mathrm{BH}$ radius becomes smaller than the Planck scale so that the (semi)classical analysis of gravity breaks down and hence we do not consider such a case. We here assume that if $\Gamma_{\mathrm{H}}<\Gamma_{\mathrm{D}}$ holds at $M_{+}=1$, the Planck-mass $\mathrm{BH}$ catalyzes the phase transition to the AdS vacuum.

We can see how the presence of $\mathrm{BH}$ changes the vacuum decay rate by comparing it with the CdL tunneling rate. In the thin-wall approximation, the bounce action is 


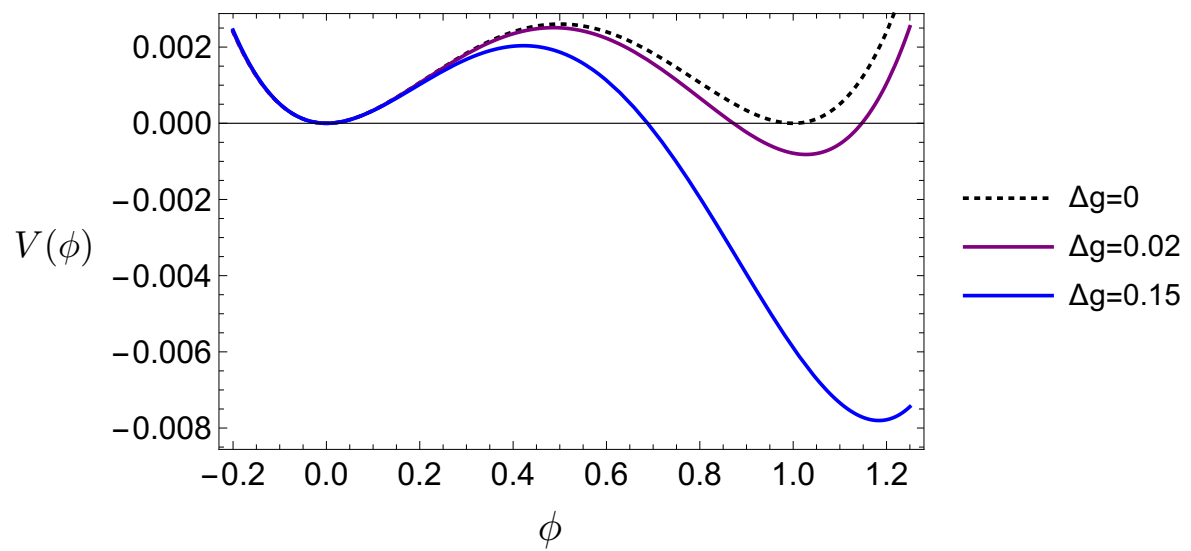

Figure 3. Potential shape for $m=1 / 2 \sqrt{3}, \lambda=1$. The dashed line represents $\Delta g=0$ for which the two vacua degenerate, and the potentials with the purple and blue lines lead to a thin-wall and thick-wall bubbles, respectively.

evaluated as [14]

$$
B_{\mathrm{CDL}}=\frac{\pi \bar{\sigma}^{4} l^{6}}{16 G\left(1-\bar{\sigma}^{2} l^{2} / 4\right)}
$$

The bounce action around a small BH eq. (2.43), which is of our interest as seen in the above, is less than the CDL bounce action for $1<M_{+} \ll \alpha^{-1}$.

\subsection{Connecting the parameters of thin-wall bubbles with the effective po- tential}

The analysis of thin-wall bubbles with Israel junction condition uses some parameters characterizing a vacuum bubble, e.g. $\sigma$ and $l$ in the previous subsection. These parameters include some details of an effective potential of scalar field. We here clarify the relation between the thin-wall parameters and the effective potential. Let us consider the following toy potential of scalar field,

$$
V(\phi)=\frac{m^{2}}{2} \phi^{2}-\sqrt{\frac{\lambda}{6}} \frac{g m}{3} \phi^{3}+\frac{\lambda}{4 !} \phi^{4} .
$$

It has a true vacuum at $\phi_{\mathrm{tv}} \simeq 2 \sqrt{3} \mathrm{~m} / \sqrt{\lambda}$ for $g>g_{0}=3 / \sqrt{2}$ with $V\left(\phi_{\mathrm{tv}}\right)=-4 \sqrt{2}\left(\mathrm{~m}^{4} / \lambda\right) \Delta g$ to the first order in $\Delta g \equiv g-g_{0}$, and the false vacuum at the origin $\phi_{\mathrm{fv}}=0$ with $V\left(\phi_{\mathrm{fv}}\right)=0$. The potential maximum between true and false vacua is given by $V_{\text {barrier }}=(3 / 8) \mathrm{m}^{4} / \lambda$. Thus $V_{\text {barrier }} \gg \Delta V$ is satisfied for $\Delta g \ll \sqrt{3+\sqrt{3}}-3 / \sqrt{2} \simeq 0.05$, in which case the thinwall approximation is appropriate, see figure 3 and eq. (2.23). In the following analysis, we use the potential eq. (2.48) with $(m, g, \lambda)$ being independent parameters that fixes the potential.

Next let us evaluate the thin-wall parameters and results in terms of the potential parameters with $V(\phi)$ eq. (2.48). The tension and the AdS radius are given by

$$
\bar{\sigma}=\frac{16 \pi m^{3}}{\lambda}, \quad l=\sqrt{\frac{3}{32 \sqrt{2} \pi}} \sqrt{\frac{\lambda}{\Delta g}}\left(\frac{1}{m}\right)^{2} .
$$


Then we obtain $\bar{\sigma} l \sim(m / \sqrt{\lambda}) / \sqrt{\Delta g} \sim \phi_{\mathrm{tv}} / \sqrt{\Delta g}$, and the condition $\bar{\sigma} l \ll 1$ is satisfied for sub-Planckian false vacuum $\phi_{\mathrm{tv}} \ll 1$ with $\Delta g \sim \mathcal{O}(0.1)$. In ref. [27] it is argued that for $\bar{\sigma} l>1 / 2$ there is no static $O(3)$ bubble solution, which should satisfy $f_{ \pm} \dot{\tau}_{ \pm}>0$. Now the physical meaning of this condition is clear. For $\bar{\sigma} l \geq \mathcal{O}(1)$, the two vacua are degenerated too much $\Delta g \simeq \phi_{\mathrm{tv}}^{2} \ll 1$, gravitational back reaction prevents the system from having a bounce solution.

The bounce action and solutions for $\bar{\sigma} l \ll 1$ are written as

$$
\begin{aligned}
\alpha & \simeq \frac{2 \sqrt{2}}{3} m \Delta g, \\
\mu_{-} & \simeq \begin{cases}\left(1-\frac{\sqrt{2} \pi m^{2}}{\lambda \Delta g}\right) M_{+} & \left(M_{+} \gg 1 /(2 \alpha)\right) \\
M_{+}-\frac{4 \pi m}{3 \lambda \Delta g^{2}} & \left(M_{+} \ll 1 /(2 \alpha)\right)\end{cases} \\
B & \simeq \begin{cases}\frac{8 \sqrt{2} \pi^{2} m^{2}}{\lambda \Delta g} M_{+}^{2} & \left(M_{+} \gg 1 /(2 \alpha)\right) \\
\frac{32 \pi^{2} m}{3 \lambda \Delta g^{2}} M_{+} & \left(M_{+} \ll 1 /(2 \alpha)\right)\end{cases} \\
R & \simeq \begin{cases}2 M_{+}\left(1+\frac{1}{128 m^{2} \Delta g^{2} M_{+}^{2}}\right) & \left(M_{+} \gg 1 /(2 \alpha)\right) \\
2 M_{+}\left(\frac{1}{2 \sqrt{2} m \Delta g M_{+}}-\frac{1}{4}\right) & \left(M_{+} \ll 1 /(2 \alpha)\right)\end{cases}
\end{aligned}
$$

which will be useful for the investigation in the next section. The bubble radius (eq. (2.53)) is further rewritten as

$$
R \simeq \frac{1}{\sqrt{2} m \Delta g} \quad \text { for } \quad M_{+} \ll(m \Delta g)^{-1}
$$

for the future use.

\section{Static bounce around a radiating $\mathrm{BH}$}

While the evaluation in the previous section based on refs. [27, 28] explicitly shows the amplification of the vacuum decay rate due to the seed $\mathrm{BH}$ for small mass, the bounce solution is obtained from the tree-level action. Since a BH emits the high-temperature Hawking radiation, one might expect that it stabilizes the scalar potential so that the vacuum decay rate is instead reduced [43]. In order to clarify which effect is dominant, it is straightforward to construct the bounce solution and evaluate the decay rate with the effective potential that takes into account the thermal effect from the Hawking radiation. This procedure is similar to the case of the evaluation of usual thermal phase transition and can be understood as the next-to-minimal order correction to the one in refs. [27, 28].

We here take into account the vacuum polarization effect of the Hawking radiation and calculate the bounce action with a thermal effective potential. Since we are interested in the spacetime of gravitationally collapsed BHs, which consists of the Region I and II in the Penrose diagram (figure 1), the state of quantum fields can be modeled by the Unruh vacuum state that gives the outgoing thermal radiation from the vicinity of the future horizon. Since it leads to the inhomogeneous temperature distribution around a BH, it is 
difficult to carry out the full 1-loop calculation with path integral formalism. ${ }^{3}$ Thus, we will only include the thermal mass, which would be the leading contribution, and neglect $O\left(\lambda^{2}\right)$ terms to yield the following expression [54]:

$$
\Gamma_{E}[\phi]=\int \sqrt{g_{E}} d^{4} x_{E}\left(\frac{1}{2}\left(\partial_{\mu E} \phi\right)^{2}+V(\phi)+\frac{\lambda}{4}\left\langle U\left|\phi^{2}\left(x_{E}\right)\right| U\right\rangle \phi^{2}\right)+O\left(\lambda^{2}\right),
$$

where $\left\langle U\left|\phi^{2}\left(x_{E}\right)\right| U\right\rangle$ is the vacuum polarization with the Unruh vacuum state $|U\rangle$. In order to evaluate the vacuum polarization, we adopt the renormalized one for the massless scalar field [48], which has the asymptotic form as $[48,54]$

$$
\begin{aligned}
& \left\langle U\left|\phi^{2}\left(x_{E}\right)\right| U\right\rangle= \begin{cases}\frac{1}{192 \pi^{2} M^{2}}-\frac{1}{8 \pi^{2} r^{2}} \int_{0}^{\infty} d \omega \frac{\sum_{\ell}(2 \ell+1)\left|B_{\ell}(\omega)\right|^{2}}{\omega\left(e^{\omega / T_{H}}-1\right)} & (r \sim 2 M) \\
\frac{1}{8 \pi^{2} r^{2}} \int_{0}^{\infty} d \omega \frac{\sum_{\ell}(2 \ell+1)\left|B_{\ell}(\omega)\right|^{2}}{\omega\left(e^{\omega / T_{H}}-1\right)} & (r \gg 2 M)\end{cases} \\
& \simeq \begin{cases}\frac{1}{256 \pi^{2} M^{2}} & (r \sim 2 M) \\
\frac{1}{192 \pi^{2} r^{2}} & (r \gg 2 M),\end{cases}
\end{aligned}
$$

where $\omega$ is a frequency of Hawking particle, $\ell$ is the angular mode, $M$ is the mass of the Schwarzschild $\mathrm{BH}, T_{H}=1 / 8 \pi M$ is the Hawking temperature, and $B_{\ell}(\omega)$ is defined as [55]

$$
B_{\ell}(\omega) \sim\left[\frac{\ell !^{2}}{(2 \ell) !(2 \ell+1) ! !}\right]^{2} \prod_{m=1}^{\ell}\left[1+\left(\frac{\omega}{m \kappa}\right)\right] \frac{2 \omega}{\kappa}\left(\frac{\omega}{2 \kappa}\right)^{2 \ell+1}, \quad \text { with } \quad \kappa \equiv 2 \pi T_{H} .
$$

Here we neglect the masses of Hawking particles, since at the leading order the massless approximation is a good approximation for the high temperature regime, which is the case of our interest. For the computational convenience we adopt the values at $r \gg 2 M$. This treatment is not problematic since the thermal correction of the transition rate becomes important when the $\mathrm{BH}$ temperature is higher than the inverse of bubble radius, i.e. when the bubble radius is larger than the $\mathrm{BH}$ radius. The size of bubble is governed by the typical scale of phase transition, as we will see later. Moreover, the values of the two-point function near the horizon, $1 / 256 \pi^{2} M^{2}$, is not significantly different from the values extrapolated from the asymptotic expression in eq. (3.2). In summary, the effective potential is written with the radius-dependent thermal mass as

$$
V_{\mathrm{eff}}(\phi, r) \sim V(\phi)+\frac{\lambda}{768 \pi^{2} r^{2}} \phi^{2}
$$

We here neglect the change of the spacetime caused by the vacuum bubble nucleation. This is valid when the $\mathrm{BH}$ mass inside the bubble, $\mu_{\mathrm{th}-}$, is close to the $\mathrm{BH}$ mass outside the bubble, $M_{+}$, and $G M_{+} / l \ll 1$ holds. We shall consider the case where this condition is

\footnotetext{
${ }^{3}$ In the case of the Hartle-Hawking vacuum, the full 1-loop effective potential is calculated by performing the path integral in an analogous way of the thermal field theory, with imposing the thermal periodic boundary condition on the Euclidean time. See refs. $[52,53]$ for details.
} 
satisfied. Note that at $r \gg 2 M$ the vacuum polarization eq. (3.2) is independent of the $\mathrm{BH}$ mass $M$, and hence the asymptotic behavior of the effective potential at $r \gg 2 M$ seems to be insensitive to the change of the $\mathrm{BH}$ mass. If that is so, then our calculation may be extendable to the situation where the seed BH disappears due to a phase transition [26].

So far we have considered the case where the system contains only one scalar field that experiences the phase transition. However, we can consider the case where there are many other fields, denoted as $\chi_{i}$, which couple to the scalar field, say,

$$
V_{\mathrm{int}}=\sum_{i} \frac{\lambda_{i}}{4} \chi_{i}^{2} \phi^{2}
$$

In such a case, the 1-loop effective potential is approximated as

$$
V_{\mathrm{eff}}(\phi, r) \simeq V(\phi)+\frac{\tilde{\lambda}}{4}\left\langle\hat{\chi}^{2}\right\rangle \phi^{2} \simeq V(\phi)+\frac{\tilde{\lambda}}{768 \pi^{2} r^{2}} \phi^{2}, \tilde{\lambda}=\sum_{i} \lambda_{i},
$$

where the effective coupling constant $\tilde{\lambda}$ is a parameter independent of $\lambda$ in $V(\phi)$. In particular, if the number of species coupled to $\phi$ is large, we extrapolate the above expression to $\tilde{\lambda} \gg 4 \pi$, beyond the validity of the perturbative analysis.

\subsection{Bounce solution around a radiating $\mathrm{BH}$}

Since the effective potential of the scalar field is lifted by the thermal effect, the false vacuum can be stabilized near the horizon. Therefore, it is expected that the decay rate is reduced or the bounce solution does not exist due to the stabilization. We here investigate the thermal effects on the bounce solutions and our methodology is presented below.

Here we consider the static $O(3)$ bounce solution that may give the least Euclidean action and calculate the equation of motion of the scalar field and the Einstein equations:

$$
\begin{aligned}
& f \phi^{\prime \prime}+f^{\prime} \phi^{\prime}+\frac{2}{r} f \phi^{\prime}+4 \pi r f \phi^{\prime 3}-\frac{\partial V(\phi)}{\partial \phi}-\frac{\tilde{\lambda}}{384 \pi^{2} r^{2}} \phi=0 \\
& \mu^{\prime}=4 \pi r^{2}\left(\frac{1}{2} f \phi^{\prime 2}+V(\phi)+\frac{\tilde{\lambda}}{768 \pi^{2} r^{2}} \phi^{2}\right)
\end{aligned}
$$

They are obtained by replacing the effective potential $V$ in eqs. (2.5) and (2.6) with $V_{\text {eff }}$ eq. (3.6). We then take the boundary conditions at the spatial infinity as the same as eq. (2.9). In general, the thermal correction to the potential leads to a corrected remnant mass $\mu_{\mathrm{th}-}$ and horizon radius $r_{h, \mathrm{th}}$, and the boundary condition at the horizon is expressed as

$$
\mu\left(r_{h, \mathrm{th}}\right)=\mu_{\mathrm{th}-}, \quad r_{h, \mathrm{th}}=2 \mu_{\mathrm{th}-} .
$$

The change of the bounce solution can be understood qualitatively as follows. As is well known, the bounce equation can be understood as the scalar field dynamics with $r$ being the "time" coordinate in the flipped potential $-V(\phi)$ from the horizon to infinity. Without thermal corrections, the flipped potential has two local maxima with $-V\left(\phi_{\mathrm{tv}}\right)>-V\left(\phi_{\mathrm{fv}}\right)$. The field starts to fall off from the point near the true vacuum to the false vacuum just 


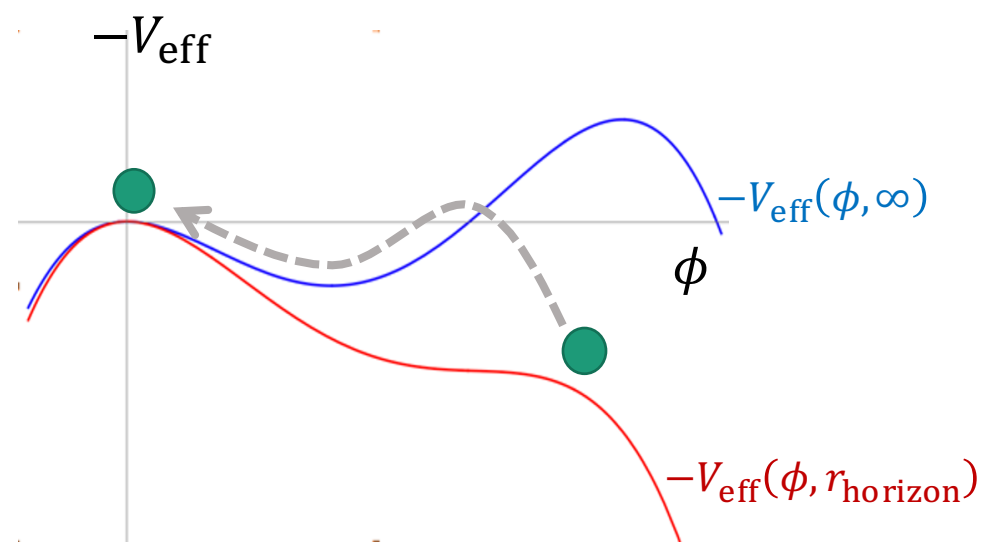

Figure 4. The Euclidean dynamics of the scalar field is shown. The red line represents the flipped effective potential at the horizon, which is stabilized by the thermal mass and the true vacuum is absent. At a distant region, the flipped effective potential is lifted as the thermal effect becomes weaker (blue line). Therefore, even if the true vacuum is absent near the horizon, a bounce solution can be constructed by using the shooting method (gray dashed line).

once $r$ deviates from the horizon. On the other hand, with thermal corrections around a sufficiently small BH, the potential is lifted around the horizon so that $-V_{\text {eff }}\left(\phi_{\mathrm{tv}}, r_{h, \mathrm{th}}\right)<$ $-V_{\text {eff }}\left(\phi_{\mathrm{fv}}, r_{h, \mathrm{th}}\right)$ with $-V_{\text {eff }}\left(\phi_{\mathrm{tv}}, r_{h, \mathrm{th}}\right)$ being no longer a local potential maximum. Thus around the horizon, we would never have the bounce solution that reaches the false vacuum. However, the thermal fluctuation becomes weaker at a distant region, and the values of $-V_{\text {eff }}$ near the true vacuum become positive so that it eventually reaches $\phi=\phi_{\mathrm{fv}}$ at infinity. The schematic picture of the evolution of the scalar field is shown in figure 4 .

To find such a solution, we numerically calculate bounce equations (3.7) and (3.8) in the shooting method. The calculation starts from the horizon $r=r_{h, \text { th }}$ with the boundary conditions,

$$
\mu\left(r_{h, \mathrm{th}}\right)=\mu_{\mathrm{th}-}=r_{\mathrm{th}-} / 2, \quad \phi_{0} \equiv \phi\left(r_{h, \mathrm{th}}\right),
$$

which determines the boundary condition for the first derivative of the scalar field at the horizon

$$
\phi^{\prime}\left(r_{h, \mathrm{th}}\right)=\frac{r_{h, \mathrm{th}} \frac{\partial V_{\mathrm{eff}}}{\partial \phi}\left(\phi_{0}\right)}{1-8 \pi r_{h, \mathrm{th}}{ }^{2} V_{\mathrm{eff}}\left(\phi_{0}\right)} .
$$

We search $\phi_{0}$ such that $\phi$ satisfies the condition eq. (2.9) at infinity by using the shooting method implemented in ref. [28] after performing the same coordinate transformation as eq. (2.13) to improve the numerical behavior near the BH horizon. Note that the bounce solution obtained from eqs. (3.7) and (3.8) is static, the bounce action $B_{\text {th }}$ is evaluated by the change of Bekenstein-Hawking entropy [27, 28]

$$
B_{\mathrm{th}}=4 \pi\left(M_{+}^{2}-\mu_{\mathrm{th}-}^{2}\right) .
$$

Performing the numerical calculations, we find that the thermal effect on the bounce solution is less significant for $\tilde{\lambda} \ll 10^{3}$. However, if we take $\tilde{\lambda} \gtrsim 10^{3}$, the bounce solution 

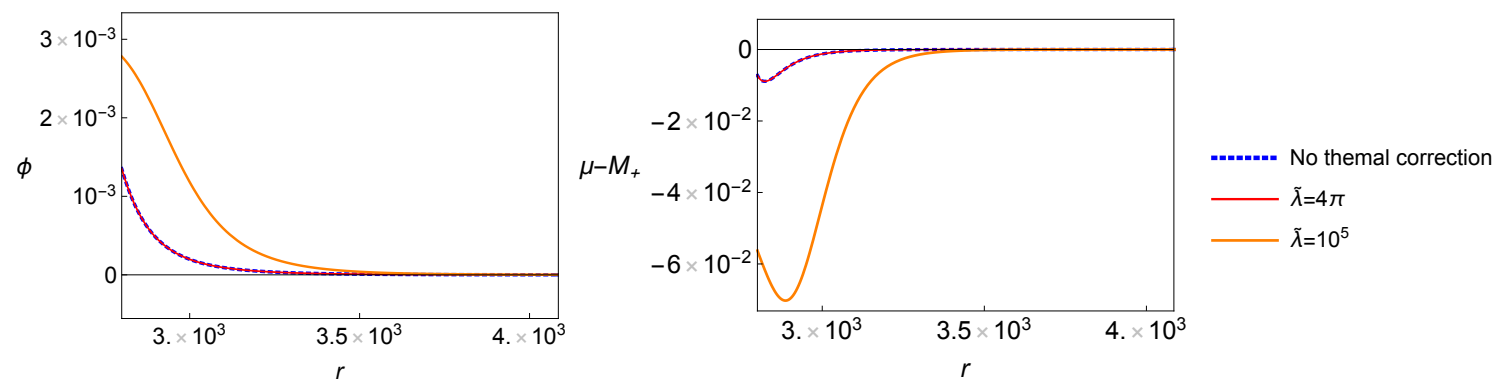

Figure 5. The bounce solutions in the case with $\lambda=4 \pi, m=2 \times 10^{-3}, g=3$ (or $\Delta g=0.88$ ) for the toy potential $V(\phi)$, and $M_{+}=1.3 \times 10^{3}$. The blue dotted line represents the bounce solution without the thermal correction. The red and orange solid line represent those with the thermal correction for $\tilde{\lambda}=4 \pi$ and $\tilde{\lambda}=10^{5}$, respectively.

and action become significantly different from the one without thermal potential, and the decay rate is sufficiently reduced. For example, in the case $m=2 \times 10^{-3}, g=3, \lambda=4 \pi$, and $M_{+}=1.4 \times 10^{3}$, we do not see the difference in the bounce solutions for $\tilde{\lambda}=4 \pi$ whereas we see the significant changes for $\tilde{\lambda}=10^{5}$, as can be seen in figure 5 . Figure 6 shows the vacuum decay rate $\Gamma_{D} \equiv \sqrt{B_{\text {th }} / 2 \pi} M_{+}^{-1} \exp \left[-B_{\text {th }}\right]$ for $m=2 \times 10^{-3}, g=3$, $\lambda=4 \pi$ as a function of $M_{+}$. The condition to ignore the effect of AdS curvature on the vacuum polarization, $M_{+} \lesssim l=1.4 \times 10^{5}$, is satisfied. Note that if the $\Gamma_{D}$ is larger than the $\mathrm{BH}$ evaporation rate $\Gamma_{H}$ (eq. (2.45)), the unwanted vacuum decay occurs before the $\mathrm{BH}$ evaporation and we would suffer from the catastrophe. We can see that without thermal corrections, the catastrophic vacuum decay for $M_{+}<10^{2}$ would be inevitable and the situation does not change for $\tilde{\lambda}<10^{4}$. If we are allowed to take extremely large $\tilde{\lambda}>10^{5}$, the thermal correction can stabilize the false vacuum state so that $\Gamma_{D}<\Gamma_{H}$ is satisfied even at $M_{+}=1$.

\subsection{Thin-wall approximation of the bounce around a radiating $\mathrm{BH}$}

The numerical calculation performed in the previous subsection implies that thermal effect on the bounce solution is not significant for $\tilde{\lambda} \lesssim 10^{3}$, regardless of the details of the other potential parameters and $\mathrm{BH}$ mass. In this subsection, in order to support this conjecture, we give an analytic investigation with the thin-wall approximation. In the thin-wall approximation, thermal correction to the bounce solution can be represented by corrections to the wall tension $\sigma$, the AdS radius $l$, and the bubble radius $R$. It is also convenient to see how the bounce equation changes due to the thermal correction, as we will see later. In the following, the subscript "th" denotes the quantities including the thermal correction.

Let us consider the case for $\bar{\sigma} l \ll 1$. In the case of $M_{+} \ll 1 /(2 \alpha)$ without the thermal correction, for which the $\mathrm{BH}$ catalysis effect is significant, the bubble radius is given as $R_{*} \simeq 2 /\left(3 \alpha_{+}\right)-M_{+} / 2 \simeq(2 / 3) \bar{\sigma} l^{2}$. The thermal correction to the potential at the bubble wall is $V_{\mathrm{th}}(\phi)=\tilde{\lambda} \phi^{2} / 768 \pi^{2} \bar{\sigma}^{2} l^{4}$. If it is much smaller than the zero-temperature potential, $\Delta V$, which holds when $\tilde{\lambda}$ is sufficiently small, the bounce configuration as well as the wall tension do not change significantly. On the other hand, the remnant BH mass slightly 


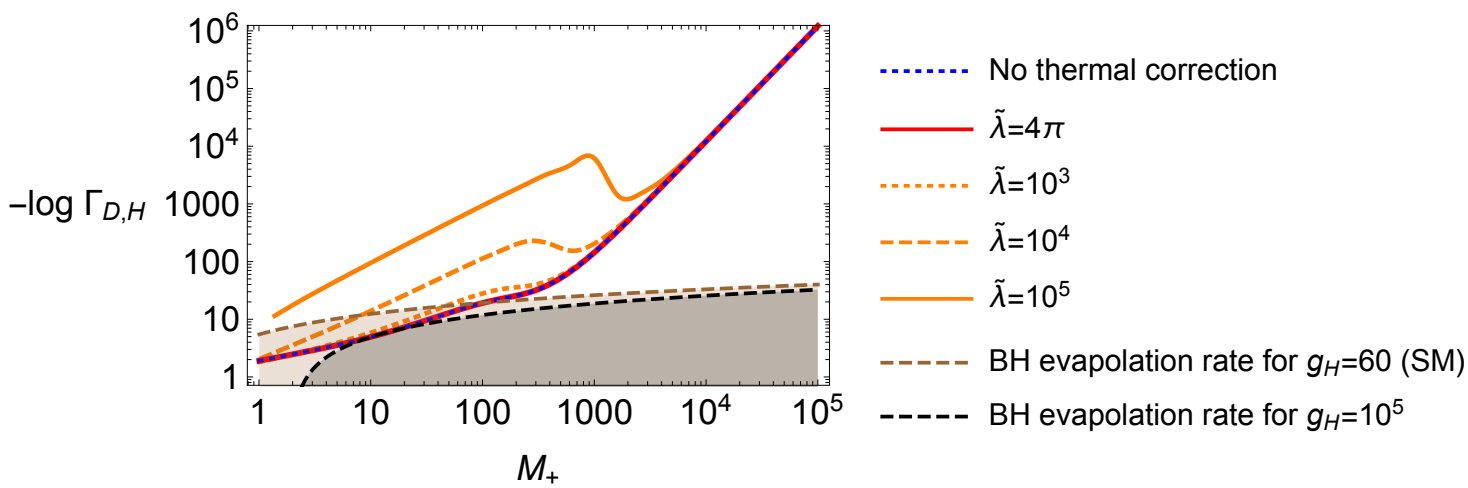

Figure 6. The vacuum decay rate as a function of $M_{+}$in the case with $\lambda=4 \pi, m=2 \times 10^{-3}$, and $g=3$ (or $\Delta g=0.88$ ) for the toy potential $V(\phi)$ (eq. (2.48)) is shown. The blue dotted line is the decay rate without the thermal correction. The decay rates including thermal corrections with $\tilde{\lambda}=4 \pi$ (red solid), $10^{3}$ (orange dotted), $10^{4}$ (orange dashed), and $10^{5}$ (orange solid) are plotted. The orange lines represent the case where there are many particles interacting with the scalar field. For comparison, the evaporation rates with $g_{H}=60$ (brown dashed) and $g_{H}=10^{5}$ (black dashed) are also shown. The former one corresponds to the case of the SM. If a line of $\Gamma_{D}$ comes below that of $\Gamma_{H}$ (shaded regions), a phase transition would occur before the $\mathrm{BH}$ evaporates.

changes as well as the bounce action. This contribution can be estimated by examining the change of the bounce equation for $\mu$, see eqs. (2.6) and (3.8). With the same field configuration for $\phi\left(\phi=\phi_{\mathrm{tv}}\left(r_{h}<r<R\right)\right.$ and $\left.\phi=\phi_{\mathrm{fv}}(r>R)\right)$, we obtain

$$
\mu_{\mathrm{th}}^{\prime}-\mu^{\prime}=\frac{\tilde{\lambda}}{192 \pi} \phi^{2} .
$$

Integrating this equation from horizon to infinity, we obtain the horizon radius of the remnant $\mathrm{BH}$ and bounce action with the thermal correction as

$$
\begin{gathered}
\mu_{\mathrm{th}-} \sim \mu_{-}+\frac{\tilde{\lambda}}{192 \pi} \phi_{\mathrm{tv}}^{2} R \simeq \mu_{-}+\frac{\tilde{\lambda} m}{16 \sqrt{2} \pi \lambda \Delta g}, \\
B_{\mathrm{th}} \sim B+\frac{\mu_{-} \tilde{\lambda}}{24} \phi_{\mathrm{tv}}^{2} R \simeq B\left(1+\frac{3 \tilde{\lambda} \Delta g}{64 \sqrt{2} \pi^{2}}\right),
\end{gathered}
$$

where we have used the explicit form of $\phi_{\mathrm{tv}}$ and eq. (2.54) to obtain the right hand side of eqs. (3.14) and (3.15). The condition that the thermal correction of the potential at the bubble wall is less significant can be expressed as

$$
\tilde{\lambda} \ll \frac{128 \sqrt{2} \pi^{2}}{\Delta g} \simeq 2 \times 10^{4}\left(\frac{0.1}{\Delta g}\right) .
$$

We can see that as long as the constraint eq. (3.16) is satisfied, the correction on the bounce action eq. (3.15) is at most order of the unity. This is consistent with figure 6 where the significant change in the bounce action is seen only for $\tilde{\lambda} \gg 10^{3}$, although the thin-wall approximation does not hold for the parameters in figure 6 . In particular, for $\tilde{\lambda} \lesssim 4 \pi$, we find that the thermal effect cannot be significant to change the vacuum decay rate. 
For $M_{+} \gg 1 /(2 \alpha)$ the bubble wall nucleates near the horizon when we do not take into account the thermal correction. The thermal effect at the true vacuum near the horizon is $V_{\mathrm{th}}\left(\phi_{\mathrm{tv}}\right) \simeq \tilde{\lambda} \phi_{\mathrm{tv}}^{2} / 3072 \pi^{2} M_{+}^{2}$ and is small, once more, when the $\tilde{\lambda}$ is sufficiently small, $\tilde{\lambda} \ll 3072 \pi^{2} M_{+}^{2} \Delta V / \phi_{\mathrm{tv}}^{2}$. In this case the bounce configuration does not change significantly. Since the thermal correction is important only near the horizon and it does not affect the wall configuration, the slight change of the system in the thin-wall approximation can be absorbed in the change of the true vacuum energy density, $\delta V=V_{\mathrm{th}}(\phi) \simeq \tilde{\lambda} \phi^{2} / 3072 \pi^{2} M_{+}^{2}$. Therefore, the thermal correction mainly changes the AdS radius, $l \rightarrow l+\delta l$, with

$$
\frac{\delta l}{l}=\frac{\tilde{\lambda} \phi_{\mathrm{tv}}^{2} / 3072 \pi^{2} M_{+}^{2}}{2 \Delta V},
$$

which changes the bounce action $B \rightarrow B+\delta B$ with $\delta B / B=2 \delta l / l$ (see eq. (2.40), which reads $\left.B \propto l^{2}\right)$. With the toy potential eq. (2.48), it is expressed as

$$
B_{\text {th }} \sim B\left[1+\frac{\tilde{\lambda} \Delta g}{288 \sqrt{2} \pi^{2}}\left(\frac{1 / 2 \alpha}{M_{+}}\right)^{2}\right] .
$$

Note that the condition that the bounce configuration does not change much is given by

$$
\tilde{\lambda} \ll 288 \sqrt{2} \pi^{2} \Delta g^{-1}\left(\frac{M_{+}}{1 / 2 \alpha}\right)^{2} \simeq 4 \times 10^{4}\left(\frac{0.1}{\Delta g}\right)\left(2 \alpha M_{+}\right)^{2} .
$$

This is also consistent with figure 6 , which shows that the deviation of the bounce actions $B$ and $B_{\text {th }}$ starts at $M_{+} /(1 / 2 \alpha) \simeq 10^{1 / 2}$ and 10 for $\tilde{\lambda}=10^{4}$ and $10^{5}$, respectively.

We find the analytic expressions that work well even when the thin-wall approximation is not valid. We performed a numerical calculation based on the shooting method in order to find a thick-wall bounce solution. Figure 7 shows the bounce action with and without the thermal correction as a function of $M_{+}$. We set $\lambda=\tilde{\lambda}=4 \pi$ and $m=1.45 \times 10^{-5}$, and perform the numerical calculation for the thin-wall $(\Delta g=0.04)$ and thick-wall $(\Delta g=0.88)$ cases. For comparison, we also plot the analytic formulae of the Euclidean action, derived in the thin-wall approximation, in figure $7 \mathrm{a}$ and $7 \mathrm{~b}$ (see eqs. (2.52), (3.15), and (3.18)). The analytic formulae are consistent with the numerical results and the thermal correction do not give significant differences in the bounce actions for $\lambda=\tilde{\lambda}=4 \pi$. The increments of bounce actions due to the thermal correction, $\Delta B$, are too small to see in figure $7 \mathrm{a}$ and $7 \mathrm{~b}$. Figure $7 \mathrm{c}$ and $7 \mathrm{~d}$ plot $\Delta B / B$ for the thin-wall and thick-wall cases, respectively, and both are well consistent with the formulae derived by the thin-wall analysis presented in eqs. (3.15) and (3.18). Therefore, we conclude that the thermal correction cannot rescue the Universe from the unwanted vacuum decay catalyzed by the $\mathrm{BH}$ unless the effective coupling to the Hawking particles, $\tilde{\lambda}$, is extremely large (eqs. (3.16) and (3.19)).

\section{Implication to the Higgs instability}

As an application of our findings in the previous section, we investigate the SM Higgs vacuum instability catalyzed by a BH. It has been studied in ref. [28] without thermal correction, which suggests that we suffer from the vacuum decay from the electroweak 
(a)

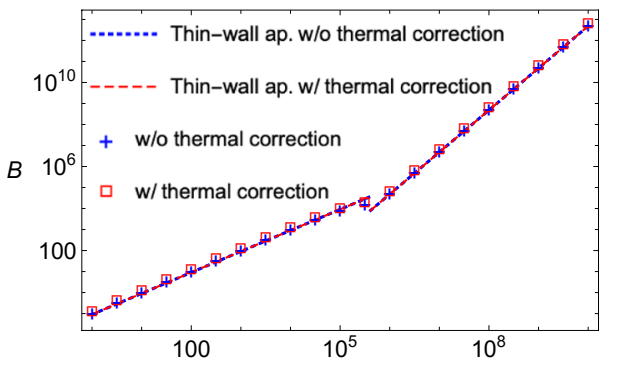

(c)

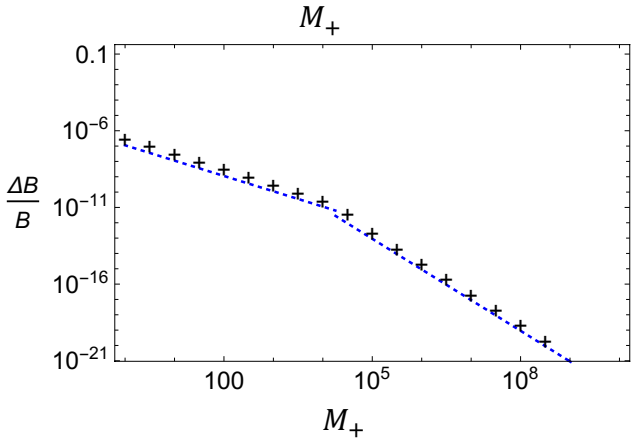

(b)

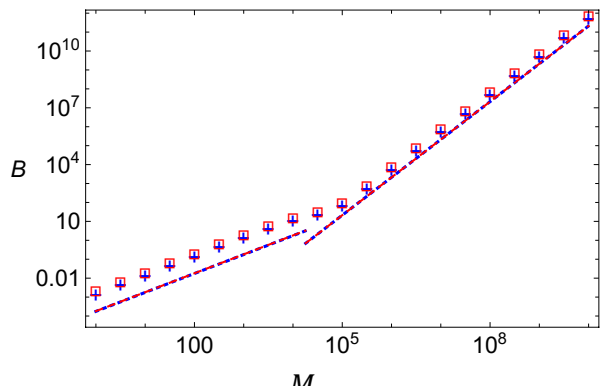

(d)

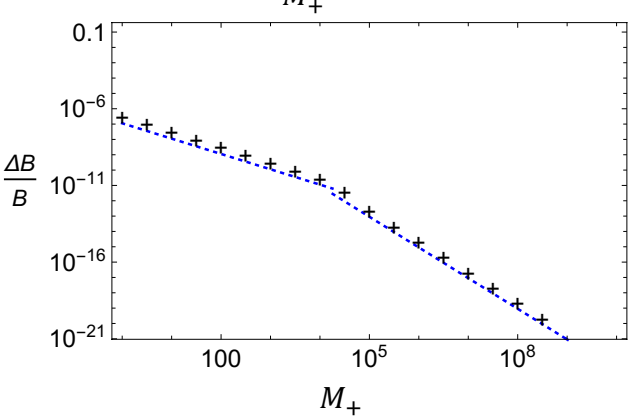

Figure 7. Figures (a) and (b) show the bounce actions as functions of seed $\mathrm{BH}$ mass with the thin-wall $(\Delta g=0.04)$ and thick-wall $(\Delta g=0.88)$ cases, respectively. We set $\lambda=\tilde{\lambda}=4 \pi$ and $m=1.45 \times 10^{-5}$. The approximate analytic formulae of the Euclidean action with/without the thermal correction (red dashed/blue dotted) are also plotted in (a) and (b). For the explicit forms of the analytic formulae, see eqs. (2.52), (3.15) and (3.18). The values of numerically obtained Euclidean actions with and without the thermal correction are plotted with the blue crosses and red squares, respectively. The differences of the bounce actions with and without the thermal correction are shown in (c) and (d) with the black crosses, along with the thin-wall approximation formulae (eqs. (3.15) and (3.18)) with the blue dotted lines.

vacuum to the AdS true vacuum, which is catalyzed by a BH with a mass $M_{+} \lesssim 10^{7}$ if the Standard Model Higgs potential is negative at the field values larger than $10^{11} \mathrm{GeV}$. We here take into account the thermal correction to see if it can rescue our Universe from this catastrophe.

The SM Higgs potential at field values much larger than the electroweak scale is well described by

$$
V_{\text {eff }}^{h}(\phi)=\frac{\lambda_{h}(\phi)}{4} \phi^{4}
$$

in the Unitary gauge. Here $\phi$ denotes the SM Higgs field and $\lambda(\mu)$ is the running Higgs quartic coupling. Instead of solving the renormalization group equations for $\lambda_{h}$ from the electroweak to high energy scales [10, 11], we here adopt the fitting formula used in ref. [28]

$$
\lambda_{h}(\mu)=\lambda^{*}+b\left(\log \left(\frac{\mu}{M_{\mathrm{pl}}}\right)\right)^{2}+c\left(\log \left(\frac{\mu}{M_{\mathrm{pl}}}\right)\right)^{4} .
$$

Here we adopt $\lambda^{*}=-0.013, b=1.3 \times 10^{-5}, c=1.7 \times 10^{-6}$ which models the Higgs potential with the running coupling for top mass $m_{t}=173 \mathrm{GeV}$ and Higgs mass $m_{h}=125 \mathrm{GeV}$. For 
these parameters, the Higgs potential becomes negative at $\phi=\phi_{c} \sim 10^{10} \mathrm{GeV} \sim 10^{-9}$ in the Planck unit.

We now study whether the potential correction from thermal fluctuation can stabilize the false vacuum enough to prevent the vacuum phase transition. It is complicated to calculate a 1-loop effective potential of a scalar field in the Schwarzschild background when it interacts with fermions or gauge fields. Thus we instead approximate the correction by the thermal mass for the SM Higgs $[11,56]$,

$$
m_{T}^{2} \sim\left(\frac{3}{16} g_{2}^{2}+\frac{1}{16} g_{Y}^{2}+\frac{1}{4} y_{t}^{2}+\frac{1}{2} \lambda_{h}\right) T^{2}
$$

with $T$ being replaced by the " $r$-dependent Hawking temperature", $T_{H}=1 / 4 \pi r$, see eq. (3.4). Here $g_{2}, g_{Y}$, and $y_{t}$ are the $\mathrm{SU}(2)_{L}, \mathrm{U}(1)_{Y}$ gauge couplings, and the top yukawa coupling, respectively. Note that the contributions from other particles are negligibly small. Let us choose the renormalization scale for the coupling constants as the Planck scale since we are interested in the phase transition induced by a tiny $\mathrm{BH}$ whose Hawking temperature is close to the Planck scale. Then we get $(3 / 16) g_{2}^{2}+(1 / 16) g_{Y}^{2}+(1 / 4) y_{t}^{2}+(1 / 2) \lambda_{h} \simeq 0.092$ and the effective potential of Higgs field around a $\mathrm{BH}$ is estimated as

$$
V_{\mathrm{eff}}^{h}(\phi ; r) \sim V_{\mathrm{eff}}^{h}(\phi)+\frac{0.092}{32 \pi^{2} r^{2}} \phi^{2},
$$

which corresponds to the effective coupling $\tilde{\lambda} \simeq 2.2$ for which the thermal correction is negligible.

With this effective potential, we solve the bounce equations in the same way as discussed in section 3, see eqs. (3.7) and (3.8). Figure 8 shows the numerical results for the bounce action. We can see that the thermal correction does not change the phase transition rate practically and the bubble nucleation rate is larger than the $\mathrm{BH}$ evaporation rate for smaller BH mass with $M_{+} \lesssim 10^{7}$, which means that the Higgs vacuum decay would be inevitable if microscopic BHs exist in the Universe. ${ }^{4}$ Note that the bounce action is proportional to the seed $\mathrm{BH}$ mass $M_{+}[28]$, similar to the case in the thin-wall approximation for the small $M_{+}$(eq. (2.44)). At $M_{+} \simeq 10^{7}$, the bounce action is of the order of unity and we do not have the exponential suppression in the vacuum decay rate for smaller BH mass.

This behavior can be understood in a similar way to the previous sections. In order for the thermal effect to be significant enough to change the bounce action, the thermal correction at a bubble wall should be comparable to or larger than the zero-temperature potential energy density. Since the zero-temperature potential is roughly described by a scale invariant potential, $V=-\left|\lambda_{h}\right| \phi^{4} / 4$ with $\lambda_{h} \sim-0.01$, the $O(4)$ bounce solution are found to be scale invariant [8], and the scale of the weak violation of the scale invariance determines the minimum bounce solution that gives the minimum bounce action. Since now we are working on the $O(3)$ bounce solution with a typical scale $\phi_{c}$, we expect that the field value inside the bubble that gives the dominant contribution for the vacuum decay is roughly $\phi_{0} \sim \phi_{c}$. Then from the balance between the bulk and wall bounce action,

\footnotetext{
${ }^{4}$ One way around would be to lower the Planck scale by the order of $10^{-7}$ so that the phase transition rate is well suppressed at the energy scales where the semi-classical approximation is valid.
} 


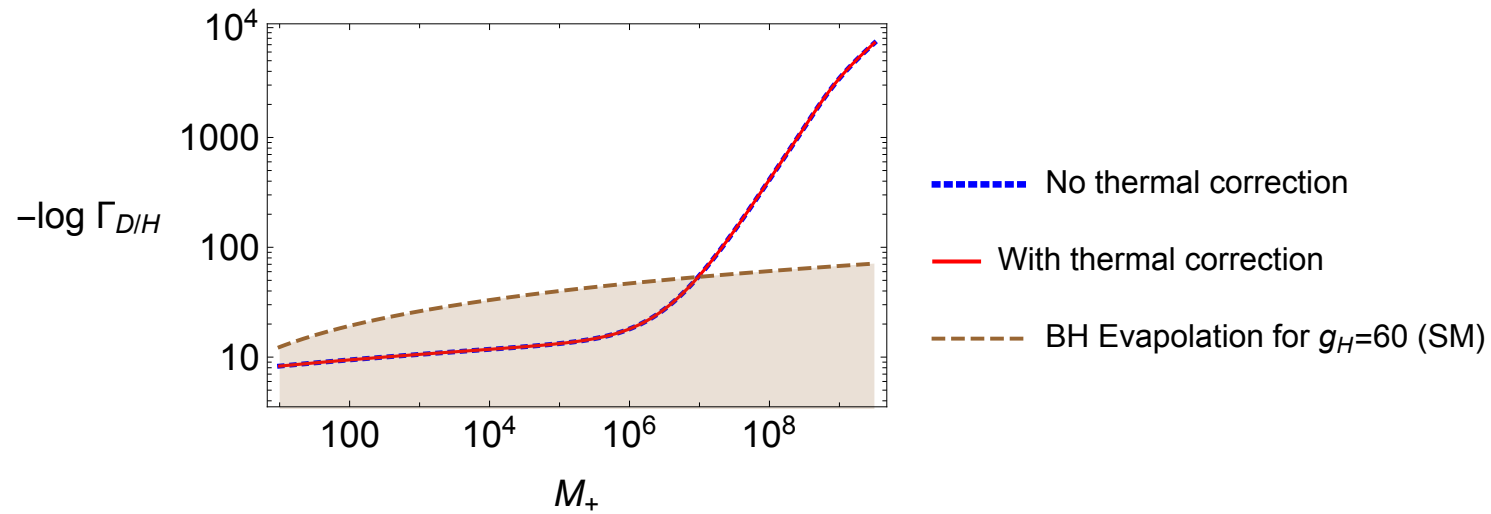

Figure 8. The bubble nucleation rate for the SM Higgs field with a $\mathrm{BH}$ as a function of the seed $\mathrm{BH}$ mass $M_{+}$is shown. Here we take the potential parameters as $\lambda^{*}=-0.013, b=1.3 \times 10^{-5}, c=1.7 \times$ $10^{-6}$. The blue dotted line indicates the bubble nucleation rate without thermal correction, while red solid line indicates the one with the thermal correction (eq. (4.4)). Brown dashed line represents the $\mathrm{BH}$ evaporation rate. The bubble nucleation rate exceeds the $\mathrm{BH}$ evaporation rate in the brown region, which means that the Universe would undergo the Higgs vacuum decay for $M_{+}<10^{7}$.

the bubble radius is roughly estimated as $R \sim 1 / \sqrt{\left|\lambda_{h}\right|} \phi_{c}$ (see eq. (2.22)). As a result, the thermal effect of the potential at the wall is estimated as $\tilde{\lambda}\left|\lambda_{h}\right| \phi_{c}^{4} / 768 \pi^{2}$, whereas the negative vacuum energy inside the bubble is estimated as $-\left|\lambda_{h}\right| \phi_{c}^{4} / 4$. Thus the thermal effect is strong enough to change the bounce configuration if $\tilde{\lambda} \gg 192 \pi^{2} \simeq 2 \times 10^{3}$. The Higgs potential, leading to the bubble configuration, can be modeled by our toy model with $\lambda \sim\left|\lambda_{h}\right|, \phi_{\mathrm{tv}} \sim \phi_{c}$, and $\Delta g \sim O(1)$. Actually, the condition for having the strong thermal effect is consistent with that derived in our toy model shown in eq. (3.16). Note that in this configuration, the AdS radius is given as $l \sim \sqrt{1 /\left|\lambda_{h}\right| \phi_{c}^{4}}$ whereas the wall tension is estimated as $\bar{\sigma} \sim\left|\lambda_{h}\right|^{1 / 2} \phi_{c}^{3}$. From eq. (2.44), we evaluate the bounce action as $B \sim \phi_{c} M_{+} /\left|\lambda_{h}\right|^{1 / 2}$, which is consistent with the numerical result (also see ref. [28]).

One might consider that the thermal correction can rescue the Universe from the unwanted vacuum decay if there are sufficiently large number of hidden fields that couple to the Higgs field to give a large effective coupling $\tilde{\lambda}$. However, such fields easily change the renormalization group equation running for the Higgs quartic coupling $\lambda_{h}(\mu)$, whose effect is much more significant than the thermal effect we consider in this work. Therefore we conclude that the $\mathrm{BH}$ catalysis effect on the vacuum decay is inevitable for the SM Higgs potential. Our very existence suggests that even a tiny primordial BH with the mass smaller than $\sim 10^{7}$ had never created in the observable Universe. As other possibilities, the SM Higgs potential may be stabilized by a new physics or is just stable with a relatively light top mass [57-59].

\section{Conclusions and discussion}

It has been discussed that a small $\mathrm{BH}$, whose mass is smaller than $M_{+}<10^{7}$, catalyzes the bubble nucleation in a false vacuum of the Higgs field and its decay rate is evaluated by the bounce action with the zero-temperature potential [26-28] and that the rate can be significantly higher than the $\mathrm{CdL}$ decay rate. However, such a small $\mathrm{BH}$ has its high 
Hawking temperature, $T_{\mathrm{H}} \gtrsim 10^{12} \mathrm{GeV}$, for which the effective potential of true vacuum is lifted to prevent the system from the vacuum decay.

In this work, we have evaluated a static $O(3)$ bounce action around a Schwarzschild $\mathrm{BH}$ in a false vacuum state. Using the Unruh vacuum state, we have taken into account a thermal mass which has its radial-dependence and is proportional to $r^{-2}$ with $r$ being the distance from the $\mathrm{BH}$. The numerical results show that the Euclidean action increases due to the thermal correction, but it is negligibly small effect for $\tilde{\lambda} \lesssim 4 \pi$. In our setup, the thermal effect can be significant when there exist a number of scalar fields couple to the metastable field and $\tilde{\lambda} \gg 10^{3}$ is satisfied. This is because even though the Hawking temperature can be high near the horizon, it is suppressed at the bubble wall and hence it cannot change the bounce configuration much. With the help of the thin-wall approximation, we have derived the formula that describes the small increase of the bounce action, which remarkably works well even for the case where the thin-wall approximation does not hold. This also gives the condition that the thermal correction can be important. That is, in order for the thermal correction to be significant, the thermal correction should be larger than the zero-temperature potential at least at the true vacuum around the bubble wall so that the bounce configuration is significantly changed. This requires an extremely large effective coupling between the scalar field and the Hawking radiation, $\tilde{\lambda} \gg 10^{4}$, since the two-point function $\left\langle U\left|\phi^{2}\right| U\right\rangle$ is suppressed by a small factor $\left(192 \pi^{2}\right)^{-1}$ (see eq. (3.2)). Note that we have assumed that the quadratic thermal mass term holds even at the true vacuum around the bubble wall. However, if the effective Hawking temperature is lower than the expectation value of the true vacuum, the thermal fluctuation is negligible as discussed in the context of the Affleck-Dine mechanism for baryogenesis [60-63]. In such a case the thermal effect would become much smaller, yet our main conclusion is unchanged that thermal effect does not alter the bounce action significantly for a reasonable coupling between the scalar field and Hawking radiation.

We have also applied our calculation to the SM Higgs vacuum instability [6-9] around a BH [28]. Thermal correction to the potential (eq. (4.4)) is estimated by the thermal mass obtained from the SM particle contents with the temperature being replaced by the $r$-dependent effective Hawking temperature. We have found out that the effective coupling between the SM Higgs and the Hawking radiation can be modeled by $\tilde{\lambda} \simeq 2.2$ in our toy model and that the thermal effect on the bounce action is negligibly small. As a result, the phase transition rate exceeds the $\mathrm{BH}$ evaporation rate for small $\mathrm{BH}, M_{+}<10^{7}$, which is the catastrophe of our Universe. Therefore we conclude that we should have never had such small primordial BHs in the observable Universe unless the SM Higgs potential is stabilized by a new physics or is stable as it is. Note that the latter possibility is still consistent with the present uncertainty of the top mass [57-59].

Finally we comment on some interpretation issue for the BH catalysis effect. Ref. [47] raised some possible interpretations for the $\mathrm{BH}$ catalysis effect. One of the interpretations is that the catalysis effect would be caused by thermal plasma around a $\mathrm{BH}$ and it can be understood as a sphaleron process in a uniform thermal bath. ${ }^{5}$ However, our study shows

\footnotetext{
${ }^{5}$ According to ref. [47], another scenario is that a vacuum bubble could be nucleated in the vicinity of the BH horizon with its non-zero kinetic energy to expand. This interpretation would work for a realistic situation where the Hawking plasma is localized near the horizon.
} 
that the thermal plasma is localized near the horizon and a nucleated bubble wall is not immersed by the plasma. Although it appears inconsistent with the proposed interpretation in [47], our argument makes sense if the catalysis effect is caused by the attractive force of a $\mathrm{BH}$ as is explained below. The size of the bubble wall is determined by the balance among the attractive force from a $\mathrm{BH}$, bubble tension, and interior vacuum energy since it is simply derived from the classical Einstein equation. Then the resulting bubble has a smaller size due to the attractive force from the $\mathrm{BH}$, which reduces the Euclidean action that is calculated by the surface integration on the Euclidean bubble. Therefore, the BH catalysis effect may be caused by the attractive force, and it is not necessary that the vacuum bubble nucleated by the $\mathrm{BH}$ catalysis effect is immersed by thermal plasma.

\section{Acknowledgments}

We thank Ruth Gregory, Keisuke Inomata, Ian Moss, Kyohei Mukaida, Davide Racco, Masaki Yamada, and Yusuke Yamada for useful discussions and comments. The work of TH is supported by Program of Excellence in Photon Science. KK is supported by JSPS KAKENHI, Grant-in-Aid for Scientific Research JP19K03842 and Grant-in-Aid for Scientific Research on Innovative Areas 19H04610. NO is supported by the JSPS Overseas Research Fellowships and the Perimeter Institute for Theoretical Physics. Research at Perimeter Institute is supported in part by the Government of Canada through the Department of Innovation, Science and Economic Development Canada and by the Province of Ontario through the Ministry of Colleges and Universities. JY is partially supported by JSPS KAKENHI Grant Nos. 15H02082, 20H00151, and Grant-in-Aid for Scientific Research on Innovative Areas 20H05248.

Open Access. This article is distributed under the terms of the Creative Commons Attribution License (CC-BY 4.0), which permits any use, distribution and reproduction in any medium, provided the original author(s) and source are credited.

\section{References}

[1] C. Caprini et al., Science with the space-based interferometer eLISA. II: Gravitational waves from cosmological phase transitions, JCAP 04 (2016) 001 [arXiv: 1512.06239] [INSPIRE].

[2] A. Mazumdar and G. White, Review of cosmic phase transitions: their significance and experimental signatures, Rept. Prog. Phys. 82 (2019) 076901 [arXiv:1811.01948] [InSPIRE].

[3] R.D. Peccei, The Strong CP problem and axions, in Lecture Notes in Physics 741, Springer (2008), pp. 3-17 [hep-ph/0607268] [INSPIRE].

[4] G.C. Branco, P.M. Ferreira, L. Lavoura, M.N. Rebelo, M. Sher and J.P. Silva, Theory and phenomenology of two-Higgs-doublet models, Phys. Rept. 516 (2012) 1 [arXiv:1106.0034] [INSPIRE].

[5] S. Kachru, R. Kallosh, A.D. Linde and S.P. Trivedi, de Sitter vacua in string theory, Phys. Rev. D 68 (2003) 046005 [hep-th/0301240] [INSPIRE].

[6] M. Sher, Electroweak Higgs Potentials and Vacuum Stability, Phys. Rept. 179 (1989) 273 [INSPIRE]. 
[7] P.B. Arnold, Can the Electroweak Vacuum Be Unstable?, Phys. Rev. D 40 (1989) 613 [INSPIRE].

[8] P.B. Arnold and S. Vokos, Instability of hot electroweak theory: bounds on $m_{H}$ and $M_{t}$, Phys. Rev. D 44 (1991) 3620 [INSPIRE].

[9] J.R. Espinosa, G.F. Giudice and A. Riotto, Cosmological implications of the Higgs mass measurement, JCAP 05 (2008) 002 [arXiv:0710.2484] [INSPIRE].

[10] G. Degrassi et al., Higgs mass and vacuum stability in the Standard Model at NNLO, JHEP 08 (2012) 098 [arXiv: 1205.6497] [inSPIRE].

[11] D. Buttazzo et al., Investigating the near-criticality of the Higgs boson, JHEP 12 (2013) 089 [arXiv: 1307.3536] [INSPIRE].

[12] S.R. Coleman, The Fate of the False Vacuum. 1. Semiclassical Theory, Phys. Rev. D 15 (1977) 2929 [Erratum ibid. 16 (1977) 1248] [INSPIRE].

[13] C.G. Callan Jr. and S.R. Coleman, The Fate of the False Vacuum. 2. First Quantum Corrections, Phys. Rev. D 16 (1977) 1762 [inSPIRE].

[14] S.R. Coleman and F. De Luccia, Gravitational Effects on and of Vacuum Decay, Phys. Rev. D 21 (1980) 3305 [INSPIRE].

[15] V. Branchina, E. Messina and M. Sher, Lifetime of the electroweak vacuum and sensitivity to Planck scale physics, Phys. Rev. D 91 (2015) 013003 [arXiv: 1408.5302] [InSPIRE].

[16] S. Chigusa, T. Moroi and Y. Shoji, State-of-the-Art Calculation of the Decay Rate of Electroweak Vacuum in the Standard Model, Phys. Rev. Lett. 119 (2017) 211801 [arXiv: 1707.09301] [INSPIRE].

[17] J. Elias-Miro, J.R. Espinosa, G.F. Giudice, G. Isidori, A. Riotto and A. Strumia, Higgs mass implications on the stability of the electroweak vacuum, Phys. Lett. B 709 (2012) 222 [arXiv:1112.3022] [INSPIRE].

[18] K. Kamada, Inflationary cosmology and the standard model Higgs with a small Hubble induced mass, Phys. Lett. B $\mathbf{7 4 2}$ (2015) 126 [arXiv:1409.5078] [INSPIRE].

[19] M. Herranen, T. Markkanen, S. Nurmi and A. Rajantie, Spacetime curvature and the Higgs stability during inflation, Phys. Rev. Lett. 113 (2014) 211102 [arXiv:1407.3141] [INSPIRE].

[20] A. Hook, J. Kearney, B. Shakya and K.M. Zurek, Probable or Improbable Universe? Correlating Electroweak Vacuum Instability with the Scale of Inflation, JHEP 01 (2015) 061 [arXiv: 1404.5953] [INSPIRE].

[21] K. Kohri and H. Matsui, Electroweak Vacuum Instability and Renormalized Higgs Field Vacuum Fluctuations in the Inflationary Universe, JCAP 08 (2017) 011 [arXiv: 1607.08133] [INSPIRE].

[22] J.R. Espinosa, D. Racco and A. Riotto, Cosmological Signature of the Standard Model Higgs Vacuum Instability: Primordial Black Holes as Dark Matter, Phys. Rev. Lett. 120 (2018) 121301 [arXiv: 1710.11196] [INSPIRE].

[23] Y. Ema, M. Karciauskas, O. Lebedev and M. Zatta, Early Universe Higgs dynamics in the presence of the Higgs-inflaton and non-minimal Higgs-gravity couplings, JCAP 06 (2017) 054 [arXiv: 1703.04681] [INSPIRE].

[24] P.J. Steinhardt, Monopole and Vortex Dissociation and Decay of the False Vacuum, Nucl. Phys. B 190 (1981) 583 [INSPIRE]. 
[25] W.A. Hiscock, Can black holes nucleate vacuum phase transitions?, Phys. Rev. D 35 (1987) 1161 [INSPIRE].

[26] R. Gregory, I.G. Moss and B. Withers, Black holes as bubble nucleation sites, JHEP 03 (2014) 081 [arXiv:1401.0017] [INSPIRE].

[27] P. Burda, R. Gregory and I. Moss, Vacuum metastability with black holes, JHEP 08 (2015) 114 [arXiv: 1503.07331] [INSPIRE].

[28] P. Burda, R. Gregory and I. Moss, The fate of the Higgs vacuum, JHEP 06 (2016) 025 [arXiv: 1601.02152] [INSPIRE].

[29] R. Gregory, I.G. Moss and N. Oshita, Black Holes, Oscillating Instantons, and the Hawking-Moss transition, JHEP 07 (2020) 024 [arXiv: 2003. 04927] [INSPIRE].

[30] D. Canko, I. Gialamas, G. Jelic-Cizmek, A. Riotto and N. Tetradis, On the Catalysis of the Electroweak Vacuum Decay by Black Holes at High Temperature, Eur. Phys. J. C 78 (2018) 328 [arXiv: 1706.01364] [INSPIRE].

[31] N. Oshita, K. Ueda and M. Yamaguchi, Vacuum decays around spinning black holes, JHEP 01 (2020) 015 [arXiv: 1909.01378] [INSPIRE].

[32] N. Oshita, M. Yamada and M. Yamaguchi, Compact objects as the catalysts for vacuum decays, Phys. Lett. B 791 (2019) 149 [arXiv:1808.01382] [INSPIRE].

[33] I. Koga, S. Kuroyanagi and Y. Ookouchi, Instability of Higgs Vacuum via String Cloud, Phys. Lett. B 800 (2020) 135093 [arXiv: 1910.02435] [INSPIRE].

[34] H. Firouzjahi, A. Karami and T. Rostami, Vacuum decay in the presence of a cosmic string, Phys. Rev. D 101 (2020) 104036 [arXiv:2002.04856] [INSPIRE].

[35] N. Oshita, Small-mass naked singularities censored by the Higgs field, Class. Quant. Grav. 37 (2020) 07LT01 [arXiv:2002.11175] [INSPIRE].

[36] S.W. Hawking, Gravitationally collapsed objects of very low mass, Mon. Not. Roy. Astron. Soc. 152 (1971) 75 [INSPIRE].

[37] B.J. Carr and S.W. Hawking, Black holes in the early Universe, Mon. Not. Roy. Astron. Soc. 168 (1974) 399 [INSPIRE].

[38] B.J. Carr, The Primordial black hole mass spectrum, Astrophys. J. 201 (1975) 1 [inSPIRE].

[39] M. Sasaki, T. Suyama, T. Tanaka and S. Yokoyama, Primordial Black Hole Scenario for the Gravitational-Wave Event GW150914, Phys. Rev. Lett. 117 (2016) 061101 [Erratum ibid. 121 (2018) 059901] [arXiv: 1603.08338] [INSPIRE].

[40] LiGO Scientific and Virgo collaborations, Observation of Gravitational Waves from a Binary Black Hole Merger, Phys. Rev. Lett. 116 (2016) 061102 [arXiv:1602.03837] [INSPIRE].

[41] D.-C. Dai, R. Gregory and D. Stojkovic, Connecting the Higgs Potential and Primordial Black Holes, Phys. Rev. D 101 (2020) 125012 [arXiv:1909.00773] [inSPIRE].

[42] K. Inomata, M. Kawasaki, K. Mukaida, T. Terada and T.T. Yanagida, Gravitational Wave Production right after a Primordial Black Hole Evaporation, Phys. Rev. D 101 (2020) 123533 [arXiv: 2003.10455] [INSPIRE].

[43] K. Kohri and H. Matsui, Electroweak Vacuum Collapse induced by Vacuum Fluctuations of the Higgs Field around Evaporating Black Holes, Phys. Rev. D 98 (2018) 123509 [arXiv: 1708.02138] [INSPIRE]. 
[44] A.D. Linde, Decay of the False Vacuum at Finite Temperature, Nucl. Phys. B 216 (1983) 421 [Erratum ibid. 223 (1983) 544] [INSPIRE].

[45] W.G. Unruh, Notes on black hole evaporation, Phys. Rev. D 14 (1976) 870 [InSPIRE].

[46] J.B. Hartle and S.W. Hawking, Path Integral Derivation of Black Hole Radiance, Phys. Rev. D 13 (1976) 2188 [INSPIRE].

[47] K. Mukaida and M. Yamada, False Vacuum Decay Catalyzed by Black Holes, Phys. Rev. D 96 (2017) 103514 [arXiv:1706.04523] [INSPIRE].

[48] P. Candelas, Vacuum Polarization in Schwarzschild Space-Time, Phys. Rev. D 21 (1980) 2185 [INSPIRE].

[49] W. Israel, Singular hypersurfaces and thin shells in general relativity, Nuovo Cim. B 44 (1966) 1 [Erratum ibid. 48 (1967) 463] [INSPIRE].

[50] J.H. MacGibbon and B.R. Webber, Quark and gluon jet emission from primordial black holes: The instantaneous spectra, Phys. Rev. D 41 (1990) 3052 [INSPIRE].

[51] J.H. MacGibbon, Quark and gluon jet emission from primordial black holes. 2. The Lifetime emission, Phys. Rev. D 44 (1991) 376 [inSPIRE].

[52] A. Flachi and T. Tanaka, Chiral Modulations in Curved Space I: Formalism, JHEP 02 (2011) 026 [arXiv: 1012.0463] [INSPIRE].

[53] A. Flachi and T. Tanaka, Chiral Phase Transitions around Black Holes, Phys. Rev. D 84 (2011) 061503 [arXiv: 1106.3991] [INSPIRE].

[54] I.G. Moss, Black Hole bubbles, Phys. Rev. D 32 (1985) 1333 [InSPIRE].

[55] D.N. Page, Particle Emission Rates from a Black Hole: Massless Particles from an Uncharged, Nonrotating Hole, Phys. Rev. D 13 (1976) 198 [InSPIRE].

[56] G.F. Giudice, A. Notari, M. Raidal, A. Riotto and A. Strumia, Towards a complete theory of thermal leptogenesis in the SM and MSSM, Nucl. Phys. B 685 (2004) 89 [hep-ph/0310123] [INSPIRE].

[57] ATLAS collaboration, Measurement of the top quark mass in the $t \bar{t} \rightarrow$ lepton + jets channel from $\sqrt{s}=8 \mathrm{TeV}$ ATLAS data and combination with previous results, Eur. Phys. J. C $\mathbf{7 9}$ (2019) 290 [arXiv: 1810.01772] [INSPIRE].

[58] CMS collaboration, Measurement of the top quark mass using proton-proton data at $\sqrt{s}=7$ and 8 TeV, Phys. Rev. D 93 (2016) 072004 [arXiv:1509.04044] [INSPIRE].

[59] F. Bezrukov and M. Shaposhnikov, Why should we care about the top quark Yukawa coupling?, J. Exp. Theor. Phys. 120 (2015) 335 [arXiv:1411.1923] [INSPIRE].

[60] A. Anisimov and M. Dine, Some issues in flat direction baryogenesis, Nucl. Phys. B 619 (2001) 729 [hep-ph/0008058] [INSPIRE].

[61] M. Fujii, K. Hamaguchi and T. Yanagida, Reheating temperature independence of cosmological baryon asymmetry in Affleck-Dine leptogenesis, Phys. Rev. D 63 (2001) 123513 [hep-ph/0102187] [INSPIRE].

[62] S. Kasuya and M. Kawasaki, Q ball formation: Obstacle to Affleck-Dine baryogenesis in the gauge mediated SUSY breaking?, Phys. Rev. D 64 (2001) 123515 [hep-ph/0106119] [INSPIRE].

[63] T. Chiba, K. Kamada, S. Kasuya and M. Yamaguchi, Fate of thermal log type Q balls, Phys. Rev. D 82 (2010) 103534 [arXiv: 1007.4235] [INSPIRE]. 